\title{
Optical ultrasound sensors for photoacoustic imaging: a narrative review
}

\author{
Bo Fu ${ }^{1,2,3}$, Yuan Cheng ${ }^{2}$, Ce Shang ${ }^{1,4}$, Jing Li ${ }^{1,4}$, Gang Wang ${ }^{2}$, Chenghong Zhang ${ }^{2}$, Jingxuan Sun ${ }^{2}$, \\ Jianguo $\mathrm{Ma}^{1,2,3}$, Xunming $\mathrm{Ji}^{5}$, Boqu $\mathrm{He}^{1,2} \wedge$ \\ ${ }^{1}$ BUAA-CCMU Advanced Innovation Center for Big Data-Based Precision Medicine, School of Engineering Medicine, Beihang University, Beijing, \\ China; ${ }^{2}$ School of Instrumentation and Optoelectronic Engineering, Beihang University, Beijing, China; Key Laboratory of Big Data-Based \\ Precision Medicine, Ministry of Industry and Information Technology, Interdisciplinary Innovation Institute of Medicine and Engineering, Beihang \\ University, Beijing, China; ${ }^{4}$ School of Biological Science and Medical Engineering, Beihang University, Beijing, China; ${ }^{5}$ Neurosurgery Department \\ of Xuanwu Hospital, Capital Medical University, Beijing, China
}

Correspondence to: Dr. Boqu He. BUAA-CCMU Advanced Innovation Center for Big Data-Based Precision Medicine, School of Engineering Medicine, Beihang University, Beijing 100191, China. Email: boquhe@buaa.edu.cn.

\begin{abstract}
Optical ultrasound sensors have been increasingly employed in biomedical diagnosis and photoacoustic imaging (PAI) due to high sensitivity and resolution. PAI could visualize the distribution of ultrasound excited by laser pulses in biological tissues. The information of tissues is detected by ultrasound sensors in order to reconstruct structural images. However, traditional ultrasound transducers are made of piezoelectric films that lose sensitivity quadratically with the size reduction. In addition, the influence of electromagnetic interference limits further applications of traditional ultrasound transducers. Therefore, optical ultrasound sensors are developed to overcome these shortcomings. In this review, optical ultrasound sensors are classified into resonant and non-resonant ones in view of physical principles. The principles and basic parameters of sensors are introduced in detail. Moreover, the state of the art of optical ultrasound sensors and applications in PAI are also presented. Furthermore, the merits and drawbacks of sensors based on resonance and non-resonance are discussed in perspectives. We believe this review could provide researchers with a better understanding of the current status of optical ultrasound sensors and biomedical applications.
\end{abstract}

Keywords: Optical ultrasound detection; photoacoustic imaging (PAI); microring resonator; Bragg grating; photoacoustic endoscopy

Submitted Jun 11, 2021. Accepted for publication Sep 23, 2021.

doi: 10.21037/qims-21-605

View this article at: https://dx.doi.org/10.21037/qims-21-605

\section{Introduction}

Photoacoustic imaging (PAI) is an indispensable method for the diagnosis and assessment of diseases, such as breast cancer (1-4), dermatology (5-8) and vascular lesions (9-11). As an interdisciplinary study of optical excitation and ultrasound detection, PAI exploits both high contrast of optical absorption and low scattering of ultrasound.
During the process of optical excitation, biological tissues are illuminated by conventional laser radiation or low-cost light emitting diodes (12-16). Photon energy is absorbed by biomolecules in the propagation path. Due to nonradiative transition, thermal energy is immediately generated and subsequently converted into ultrasonic pulses owing to thermoelastic expansion effect. The image of biological

^ ORCID: Bo Fu, 0000-0003-1409-2666; Boqu He, 0000-0002-2906-5305. 
tissues is reconstructed based on spectrally-resolved laser absorption after the detection of distributed ultrasonic pulses. It should be noted that the produced ultrasonic signals have broad angular radiation, weak pressure of several kilopascals $(\mathrm{kPa})$, and a wide frequency bandwidth ranging from several kilohertz $(\mathrm{kHz})$ to hundreds of megahertz (MHz).

Ultrasound detection plays a vital role in PAI. Conventional ultrasound transducers are based on piezoelectric materials, converting pressure information into electrical signals (17-19). Micromachined ultrasound transducers such as capacitive and piezoelectric micromachined ultrasound transducers are promising alternatives to conventional transducers (20-22). Compared with conventional transducers, micromachined ultrasound transducers are compatible with techniques of integrated circuit (23). They could suppress backward ringing effect without impedance-match layers (24). However, there still exist several inherent limitations of micromachined ultrasound transducers that hinder the applications in PAI. It was found that the performance of PAI is critically dependent on the sensitivity and bandwidth of ultrasound sensors (25). On the one hand, micromachined transducers have narrow bandwidth caused by the dimensions of the transducers (26). Thus, it is difficult for micromachined transducers to acquire multiscale information which needs sensors with high sensitivity and broad bandwidth. On the other hand, detection sensitivity is limited by decreasing the size of sensing elements (27). In this case, it is difficult to achieve high-resolution imaging based on a detector array. In addition, conventional transducers may not work precisely in strong electromagnetic fields. Moreover, ultrasound transducers based on piezoelectric films fail to achieve multi-modality imaging due to bulky packages and optical opaqueness (28).

In order to overcome these shortcomings, optical ultrasound sensors are utilized owing to high sensitivity, high spatial resolution and miniaturized capability (29-31). Optical ultrasound detection begins with freespace interferential structures and gradually evolves into fiber type with a miniaturized probe (32-34). Owing to advances in silicon photonics, integrated sensors based on silicon-on-insulator technology are developed with high sensitivity, wide detection bandwidth and miniaturized packages $(12,35)$. Nowadays, several papers have reported on optical detection of ultrasound, where typical technologies including microring resonators and fiber Bragg grating (FBG) sensors were introduced $(36,37)$.
Moreover, systematic summaries of different technologies have been presented in view of different principles of detection $(12,30,38)$. Recently, optical ultrasound sensors have attracted extensive attention due to the excellent performance and widespread applications, which is worthy of a comprehensive review.

In this review, we summarize optical ultrasound sensors based on resonance and non-resonance methods. The principles, basic characteristics, and developments of these sensors are discussed. According to the resonant and nonresonant types of sensors, we select classic and strong timeliness papers of optical ultrasound sensors. These papers include classic papers in the field, highly cited papers, and papers from well-known research groups. Besides, the state of the art of optical ultrasound sensors and biomedical applications in PAI are also presented (39). The merits and drawbacks of resonance- and non-resonance-based sensors are listed in perspectives. We believe this review could provide researchers with a better understanding of the current status of optical ultrasound sensors and applications in biomedicine.

We present the following article in accordance with the Narrative Review reporting checklist (available at https:// dx.doi.org/10.21037/qims-21-605).

\section{Properties and applications of optical ultrasound sensors}

Optical ultrasound sensors are widely used in biomedical applications owing to the miniaturization and high sensitivity. The detection sensitivity, bandwidth, and acceptance angle are three key parameters for evaluating the performance of optical ultrasound sensors $(30,40)$. Detection sensitivity is the most important factor since it directly influences the signal-to-noise ratio (SNR) of sensing process. Sensitivity is commonly quantified by the noise equivalent pressure (NEP) (41). NEP is measured as the minimum detectable pressure, which represents the noise level during signal detection. Small NEP is helpful to obtain high SNR. High detection sensitivity (Pa or sub-Pa) is required for monitoring information from deeper tissues precisely in medical applications. Detection bandwidth has a significant impact on spatial resolution in PAI. The spatial resolution includes axial and lateral resolution, which is inversely proportional to detection bandwidth. For higher resolution, the detection bandwidth of sensors should be broad enough to cover the generated photoacoustic signals. The acceptance angle represents the range that detectors 


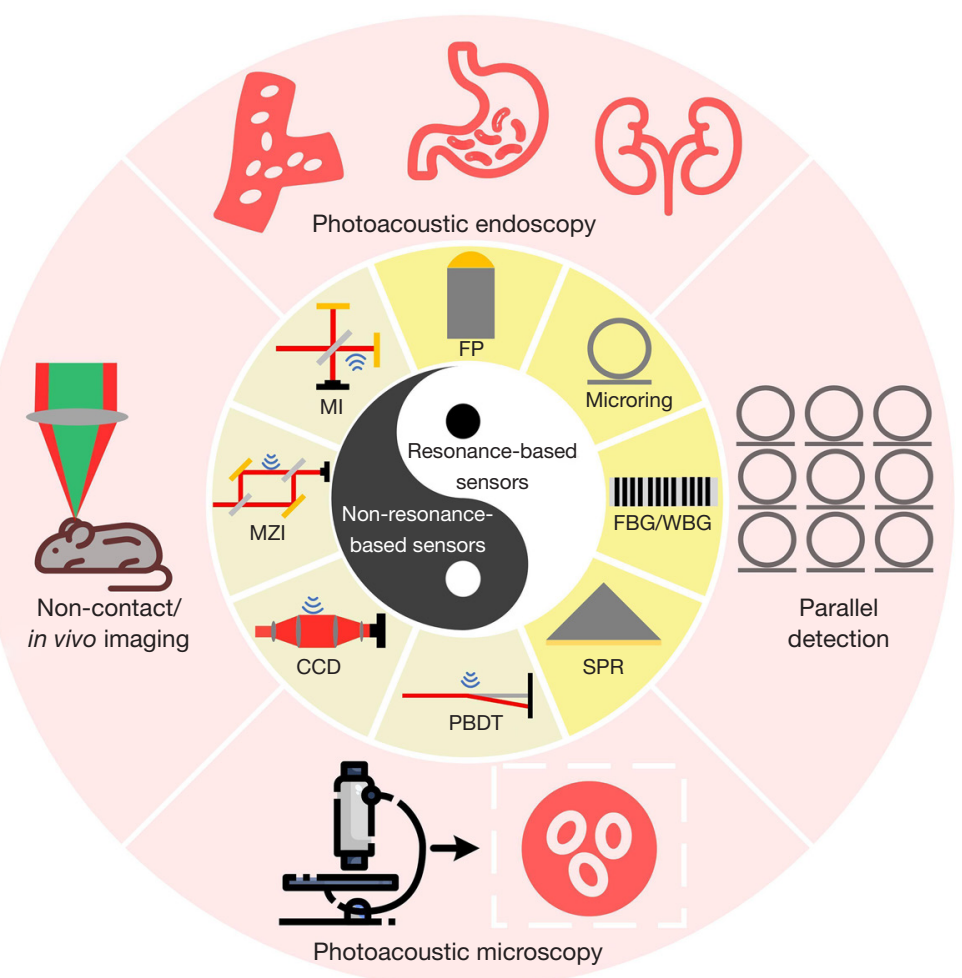

Figure 1 Classifications and applications of optical ultrasound sensors. FP, Fabry-Perot; FBG, fiber Bragg grating; WBG, waveguide Bragg grating; SPR, surface plasmon resonance; PBDT, probe beam deflection techniques; CCD, charge coupled devices; MZI, Mach-Zehnder interferometer; MI, Michelson interferometer.

could measure signals. This parameter depends on the physical dimensions and geometrical shapes of sensors, including shapes of point, disk, bar and ring. A large acceptance angle is beneficial to uniform spatial response and less image distortion.

Optical ultrasound sensors fall into two categories according to the physical principle, including resonancebased and non-resonance-based optical ultrasound sensors. Resonant sensors are specified by the resonant cavity where light repeatedly oscillates inside. Photoacoustic signal alters the length of the cavity, thus changes the transmission and reflection of the probe light. The ultrasound signals can be reconstructed by monitoring the variation of the transmittance of probe light. Instead of possessing distinct cavities, non-resonant sensors measure the beam deflection, amplitude modulation, and phase change of probe light. Figure 1 shows optical ultrasound sensors and biomedical applications. The applications of optical ultrasound sensors are discussed in this section, including photoacoustic endoscopy, non-contact imaging, in vivo imaging, photoacoustic microscopy and parallel detection $(42,43)$.

\section{Resonance-based sensors and applications}

Resonance-based optical ultrasound sensors including Fabry-Perot (FP) sensors (44-47), microring resonators (36), Bragg grating sensors (48-50) and sensors based on surface plasmon resonance (SPR). The emergence of resonancebased sensors benefits from technical advances in fiber optics and silicon photonic integration such as silicon-oninsulators $(47,49,51,52)$.

\section{FP resonators}

In 1973, Thomson et al. (53) firstly demonstrated an FP resonator to detect acoustic surface vibration. This kind of sensor has been gradually developed in terms of physical structure (54,55), compositive materials (56-59) and new technologies (60-63). FP sensors with excellent performances are suitable for photoacoustic endoscopy in medical applications $(61,64)$.

A single FP sensor has a resonant cavity that consists of a thin polymer film. The polymer film is sandwiched between two mirrors of high and partial reflectance, 


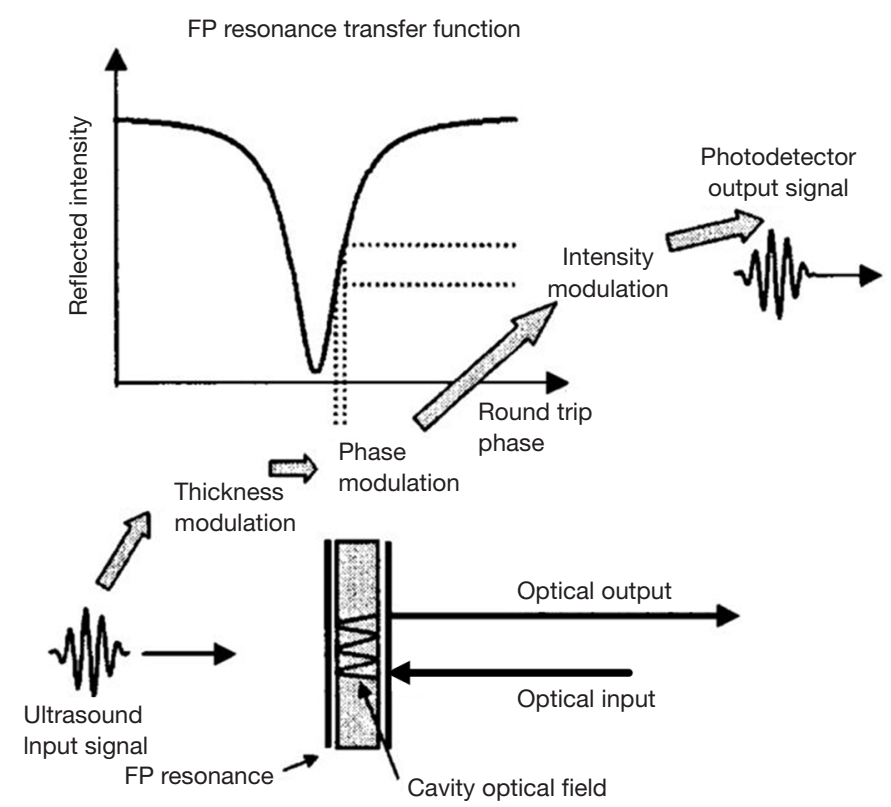

Figure 2 The basic operation principle of the FP resonator. Adapted with permission from (45). Copyright 2005 AIP Publishing. FP, FabryPerot.

respectively. The detection mechanism is schematically illustrated in Figure 2. Incident light enters the FP cavity and is reflected multiple times in the resonator. In the condition of resonance, the round-trip path length of the resonator equals an integer multiple of optical wavelength. The reflected and circulating light forms destructive interference, which is characterized by a steep dip in the reflected spectrum near the resonance wavelength. The thickness of the cavity is modulated by ultrasonic pressure. Therefore, the ultimate optical distance is modified, leading to the intensity variation of the reflected light.

The early FP resonator was in form of a spatial head with a $38-\mu \mathrm{m}$-thickness polymer film located between two dichroic mirrors, as shown in Figure $3 A(45,54,68)$. The dichroic mirrors and their coatings were specialized for exciting and interrogating laser pulses with high transmission (500-1,200 $\mathrm{nm}$ ) and high reflectivity $(1,500-1,600 \mathrm{~nm})$, respectively. To miniaturize biosensors in medical applications, optical ultrasound detection was conducted to obtain thickness changes of FP film attached to the tip of a single-mode fiber. Morris et al. (65) proposed an FP sensor based on fiber optics, which was featured with a peak NEP of $15 \mathrm{kPa}$ over a bandwidth of $20 \mathrm{MHz}$. The facet of the FP interferometer placed on fiber optics was planar, as shown in Figure 3B. The probe pulses became divergent after exiting the bound fiber core. In the cavity circulation, multiply reflected light would degrade the phase sensitivity of the interferometer. In this case, the film thickness of the planar FP sensor was fewer than $10 \mu \mathrm{m}$ and the NEP corresponded to $1 \mathrm{kPa}$. Zhang et al. (66) designed an FP fiber sensor with a concave cavity to eliminate the phase divergence and increase the sensitivity, as illustrated in Figure 3C. The NEP of this FP sensor was down to $8 \mathrm{~Pa}$ over a $20-\mathrm{MHz}$ bandwidth. In 2017, an ultrasensitive FP sensor with a $340-\mu m$-thick film created the minimum NEP to 2.6 $\mathrm{Pa}$ over a 2.8-MHz bandwidth (46). In order to solve the same problem, Ma et al. (69) proposed three unique fiber optical-based designs with an eccentric core, an absorptive shield, and an arc edge. These approaches were applied to eliminate the constructive interference, optimize the energy dissipation, and reduce the noise level. Compared with the planar FP fiber sensor, the lownoise design raised the SNR to $32.1 \mathrm{~dB}$ under the same test conditions. Figure $3 D$ showed that not only the single sensing element but the FP sensor array was designed to overcome the scale-up difficulties for resonance-based sensors (67). Eight interrogation beams at the wavelength of $1,550 \mathrm{~nm}$ were used to interrogate the target across the FP sensor. The photoacoustic signals with corresponding modulated information were thus measured by parallel processing. This imaging system achieved 3D images in fast image acquisition time of $10 \mathrm{~s}$ due to high repetition rate of 
A

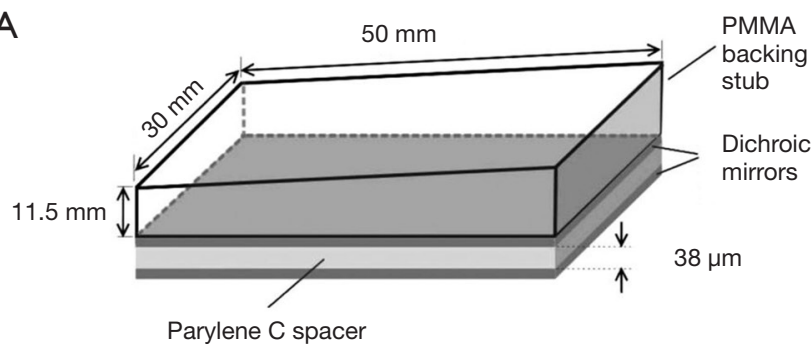

C
B

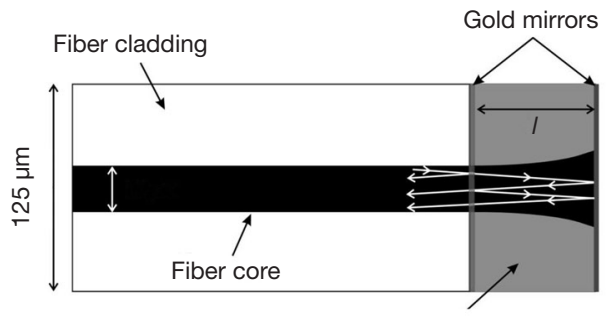

Polymer spacer (parylene C)
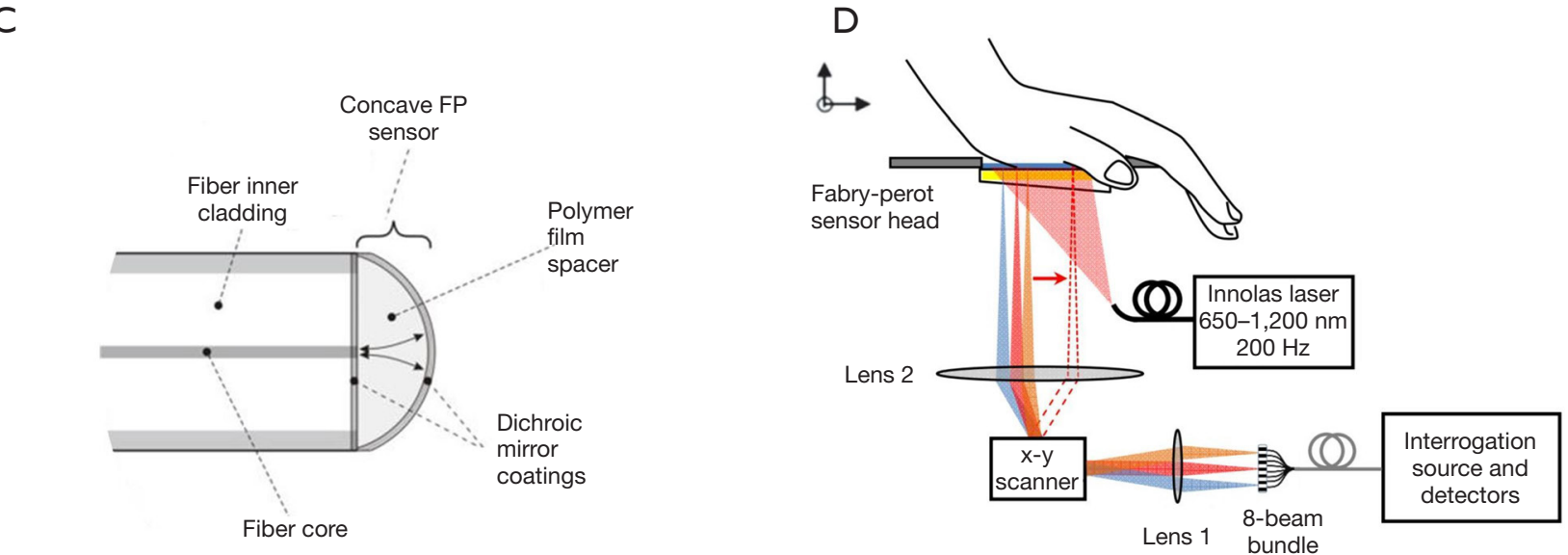

Figure 3 Various types of FP sensors and typical detecting systems employing FP sensors. (A) A spatial FP sensor head consists of a 38- $\mathrm{mm}$ polymer (Parylene C) film spacer sandwiched between two dichroic mirrors. Adapted with permission from (54). Copyright 2008 OSA Publishing. (B) A planar polymer film FP interferometer is deposited at the tip of a single-mode optical fiber. Adapted with permission from (65). Copyright 2009 Acoustical Society of America. (C) A concave polymer film FP interferometer is deposited at the tip of a singlemode optical fiber. Adapted with permission from (66). Copyright 2011 SPIE Publishing. (D) An FP interferometer array. Adapted with permission from (67). Copyright 2016 SPIE Publishing. FP, Fabry-Perot.

the excitation laser pulses.

Photoacoustic endoscopy is a fast-growing technique that realizes PAI in deep-located biological organs with miniaturized probes. Owing to the miniaturized size, fiberoptics-based FP resonators are suitable for minimally invasive imaging and photoacoustic endoscopy. It is difficult for PAI to detect organs and blood vessels due to acoustic attenuation in biological tissues. In this case, photoacoustic endoscopy can directly locate the targeted deep tissues to probe the structural and functional information, which is widely used in early-stage cancer detection of digestive $(70,71)$, respiratory, urogenital $(72,73)$ and intravascular imaging (74-76). The endogenous optical contrast of photoacoustic endoscopy can be employed to quantify the concentrations of oxyhemoglobin, deoxyhemoglobin, melanin, lipids, water and cytochromes. In 2015, Ansari et al. (77) reported on an endoscopic probe with a coherent fiber bundle, which was used to optically address different spatial points on an FP sensor located at the distal end of the bundle. The coherent fiber bundle overcomes the drawbacks of remote mechanical measurement. Moreover, this method took advantages of FP sensor, such as superior acoustic performance and practicality. In 2020, Chen et al. (61) designed a photothermally tunable FP interferometer for broadband acoustic sensing. They set up a photoacoustic mesoscopic imaging system based on the homemade sensor and obtained images of two phantoms and an ex vivo mouse kidney.

There are two viewing approaches, forward- and sideviewing, for existing photoacoustic endoscopies based on FP sensors.

Forward-viewing sensors detect the ultrasonic information in the direction of optical axis. It is desirable to introduce minimally invasive probes to internal organs such 
A

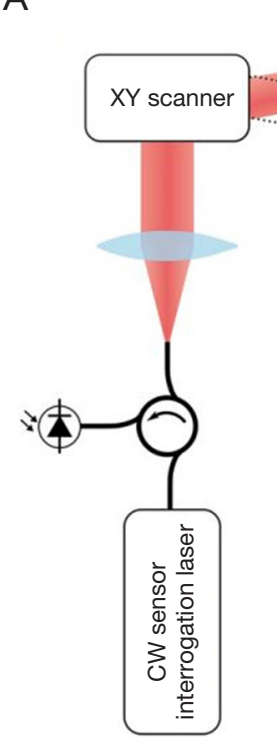

C

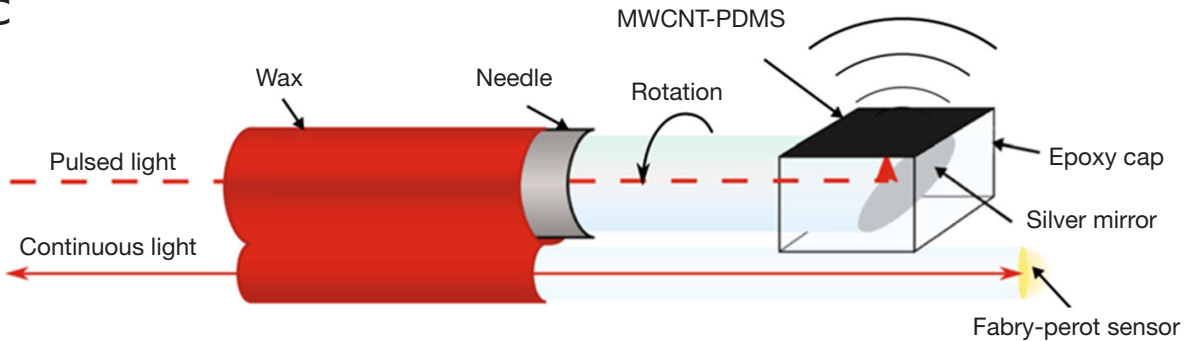

B

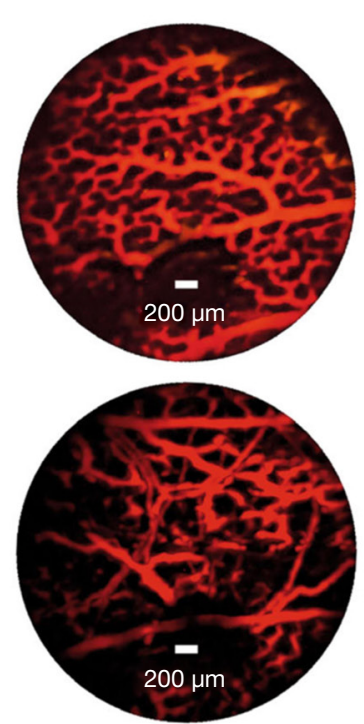

D

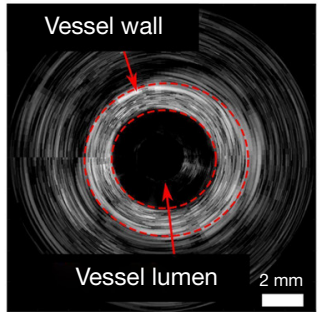

Figure 4 Medical imaging applications based on FP resonators. (A) A forward-viewing endoscopic probe based on a transparent FP polymer-film ultrasound sensor. (B) Photoacoustic images of an ex vivo duck embryo. The maximum intensity projections were for depth range from 0 to $200 \mu \mathrm{m}$ of the same embryo's two regions. (C) A side-viewing optical ultrasound probe including an FP sensor. (D) A rotational optical ultrasound image of an ex vivo swine carotid artery. (A,B) are adapted with permission from (47). Copyright 2018 Springer Nature. (C,D) are adapted with permission from (64). Copyright 2019 Springer Nature. FP, Fabry-Perot.

as animal embryos. As shown in Figure $4 A$, a transparent FP resonator attached to the end of an optical fiber bundle was employed in forwarding detection (47). The excitation laser pulses and the interrogation continuous laser were wavelength multiplexed into the fiber bundle, providing wide-field radiation and a compact structure. The corresponding photoacoustic images were captured in an ex vivo duck embryo as shown in Figure $4 B$. The excitation laser wavelength was $590 \mathrm{~nm}$ at the repetition rate of $30 \mathrm{~Hz}$ and the incident energy density was $15 \mathrm{~mJ} / \mathrm{cm}^{2}$, below the permissible exposure for biological tissues (78). The measured lateral resolution of the FP resonator ranged from 45 to $170 \mu \mathrm{m}$ for the depth between 1 and $7 \mathrm{~mm}$ with a -3-dB bandwidth of $34 \mathrm{MHz}$.
Side-viewing sensors receive the signal perpendicular to the optical axis, where cylindrical anatomical structures are feasibly mapped in pictures for assessment and analysis of arteries. Figure $4 C, 4 D$ present the schematic of an FP-sensorbased side-viewing optical ultrasound probe and intraluminal imaging of healthy swine carotid arteries, respectively (64). The excitation laser pulses were interacted with a multiwalled carbon nanotube and polydimethylsiloxane composite coating to produce the ultrasonic pressure. The distributed information in a ring geometry could be omnidirectionally monitored by a $360^{\circ}$-rotated and pointlike FP sensor, with an NEP of $100 \mathrm{~Pa}$ over a $20-\mathrm{MHz}$ bandwidth. The best axial resolution was less than $50 \mu \mathrm{m}$ at a cut-off ultrasound frequency of $20 \mathrm{MHz}$. 

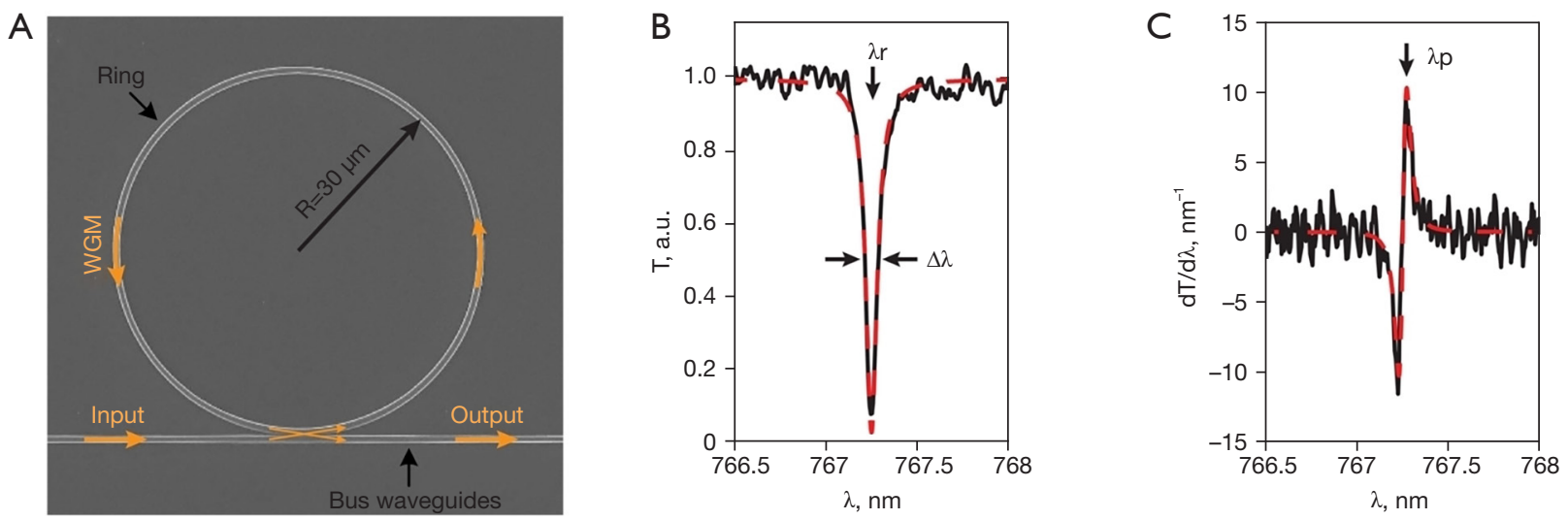

Figure 5 Geometry and operating principle of the microring resonator. Schematic of (A) a microring resonator, (B) its resonance spectrum, and $(\mathrm{C})$ ultrasound detection using the optical resonance of a microring sensor: ultrasound pressure pulse causes a dynamic shift of resonance wavelength, producing a modulated intensity in the form of a pulse in the light output. (A-C) are adapted with permission from (83). Copyright 2014 Spring Nature.

\section{Microring resonators}

Optical ultrasound sensors based on microring resonators have attracted considerable attention owing to high sensitivity, compact footprint and mass producibility (79-86). Moreover, it is more convenient for microring resonators to build detecting arrays on chip-scale packages.

As shown in Figure 5A, a typical microring resonator is composed of a straight bus waveguide and a ring waveguide, which are isolated by a low-dielectric gap. During ultrasound detection, interrogating laser pulses are injected into the microring resonator through the input port of the bus waveguide. An evanescent optical field is coupled from the bus waveguide to the ring waveguide in the interacting region of these two waveguides. Moreover, the evanescent field is circulated repeatedly in the ring geometry. Finally, a transmitted optical field is measured at the output port of the bus waveguide (83).

When the accumulated phase in the ring waveguide is equal to integral multiples of $2 \pi$ during the ultrasound detection by microring resonators, the phase difference of optical fields in bus and ring waveguides corresponds to $\pi$ because of half-wave loss (35). In this circumstance, the resonance condition is satisfied, then destructive interference occurs in the interacting region of the microring. Hence, a sharp dip appears in the transmission spectrum, which is shown in Figure 5B. The quality (Q) factor of the microring resonator can be express as $\mathrm{Q}$ $=\lambda / \Delta \lambda$, where $\lambda$ is the resonance wavelength, and $\Delta \lambda$ is the full width at half maximum of the wavelength range. The microring resonator has the physical deformation induced by ultrasonic sources (87). Subsequently, the effective refractive index $\left(\mathrm{n}_{\text {eff }}\right)$ of transmitted optical modes is immediately changed. Meanwhile, the elastic-optic effect of waveguide materials also has a contribution to the change of $\mathrm{n}_{\text {eff }}$. The amplitude of ultrasound sources, estimated by measuring transmission variation, is closely related to the shift of resonant wavelength as illustrated in Figure 5C. A narrow-band laser is usually employed as the interrogating light source and the probe wavelength is fixed at the maximum sensitivity point of the microring resonator (88).

The sensitivity of a microring sensor can be defined as $\mathrm{S}=(\mathrm{dT} / \mathrm{d} \lambda)\left(\mathrm{d} \lambda / \mathrm{dn}_{\text {eff }}\right)\left(\mathrm{dn}_{\text {eff }} / \mathrm{dP}\right)$, where $\mathrm{T}$ is the ratio of transmitted and injected optical field power in the microring resonator, $\mathrm{P}$ is the pressure of ultrasonic signals (88). The first term $\mathrm{dT} / \mathrm{d} \lambda$ can be interpreted as the slope of the relationship between transmission and wavelength, which is proportional to the $\mathrm{Q}$ factor. The second term is the wavelength shift caused by variations of $n_{\text {eff. }}$ The last term $\mathrm{dn}_{\mathrm{eff}} / \mathrm{dP}$ represents the change of $\mathrm{n}_{\text {eff }}$ induced by ultrasonic pressure. The refractive index of the polymer waveguide is associated with the deformation of waveguide. When simulated in Comsol Multip270hysics (COMSOL, Inc., Los Angeles, CA, USA), the properties of materials are counted including Young's modulus, elastic-optic coefficient and cross-section area of microring resonators. The dependence of $\mathrm{dn}_{\text {eff }} / \mathrm{dP}$ is $-6.8 \times 10^{-5} \mathrm{MPa}^{-1}$ in the reference (83). Microring resonators with a higher $\mathrm{Q}$ factor and longer wavelength are preferred to increase the sensitivity.

Microring resonators are commonly fabricated in 
A

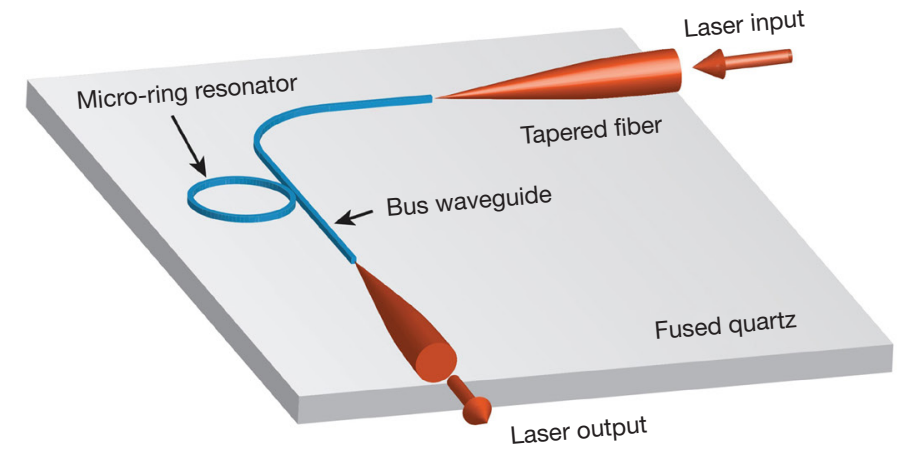

B

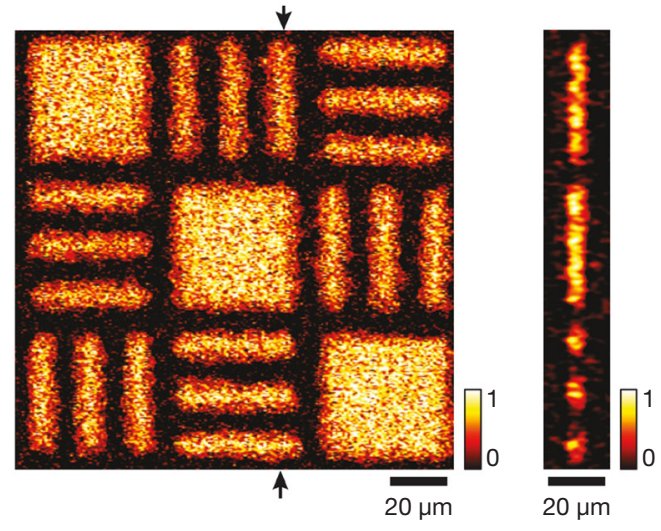

C

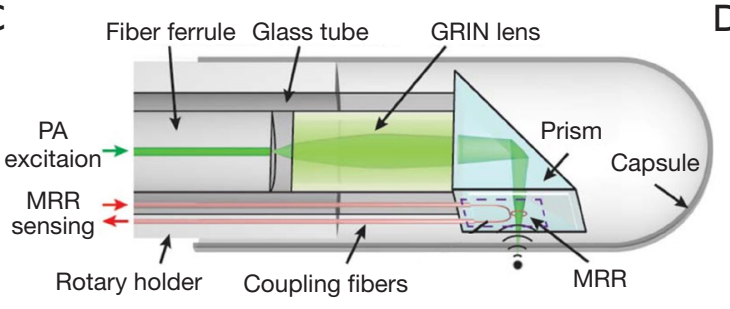

D

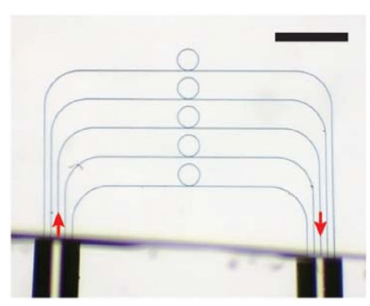

E

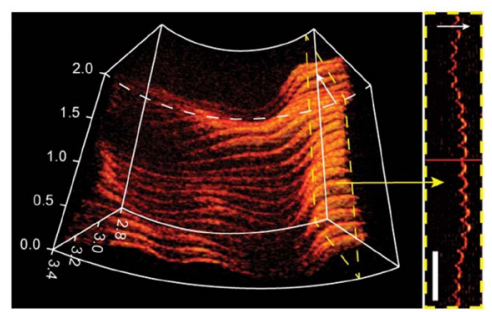

Figure 6 Schematic illustrations of the packaged microring resonator and a photoacoustic endoscopic probe, as well as experimental images. (A) Single sensing element based on the microring resonator. (B) The maximal amplitude projection image of a carbon-black thin-film target along the $x-y$ plane. (C) A microring-resonator-based photoacoustic endoscopic probe. (D) The microring resonator detecting array. (E) A volumetric photoacoustic image of a phantom test. (A,B) are adapted with permission from (83). Copyright 2014 Springer Nature. (C-E) are adapted with permission from (73). Copyright 2014 OSA Publishing.

silicon-based $(51,81,82)$ or polymer platforms $(36,79)$. The resonators based on silicon platform are made of hard materials such as silicon, silicon nitride and silicon dioxide, while others based on polymer platform use soft materials like polystyrene, polymethylmethacrylate and SU-8 $(80,83,85)$. The devices of silicon and silicon dioxide generally suffer from high losses or delocalized optical modes as the device of silicon nitride provides advantages of both high confinement and high Q factor (82). Moreover, different from the silicon device, there is no two-photon absorption at the wavelength of $1,550 \mathrm{~nm}$ for the silicon nitride device, which is desired for mid-infrared nonlinear applications. Microring resonators made of polymers are preferred for the optical detection of ultrasound. Compared with silicon, polymer withstands higher acoustic pressure and obtains higher sensitivity owing to the properties of materials.

In terms of fabrications of microring resonators, three methods are commonly used including electron beam, photo lithography and nanoimprint lithography. Among these approaches, nanoimprint lithography is suitable for polymer platform because of high-throughput production and precise dimensions. Different from electron beam and photo lithography, nanoimprinting lithography takes advantage of mechanical deformation of resist materials and overcomes the limitations of light diffraction and beam scattering (89). Therefore, nano imprinting lithography could obtain resolution without being limited by the restrictions that appears in conventional techniques. As for resolutions, patterns by nanoimprint lithography can achieve $20 \mathrm{~nm}$ (90). The electron beam lithography could realize the resolution of sub-100 $\mathrm{nm}$ (91). The photo lithography has the potential to obtain the resolution of $22-50 \mathrm{~nm}$ (92). It should be emphasized that the sidewalls of microring resonators need to be smooth, otherwise optical scattering induced by uneven sidewalls hinders the Q factor and decreases the sensitivity.

A single element microring resonator with a high $\mathrm{Q}$ factor of 10,400 is presented in Figure $6 \mathrm{~A}$ (83). The distal ports of the bus waveguide were connected to two tapered optical fibers for mode matching and light coupling. The $90^{\circ}$ bending design in the bus waveguide helped to eliminate the cross-walk effect between the input and output ports. The excellent detector had an NEP of $6.8 \mathrm{~Pa}$ 
A

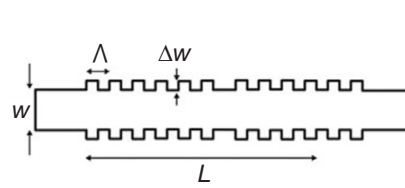

B

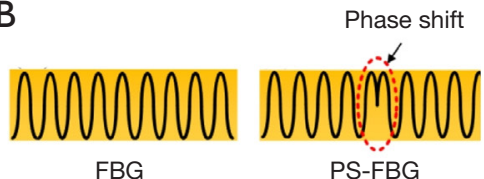

$\mathrm{D}$

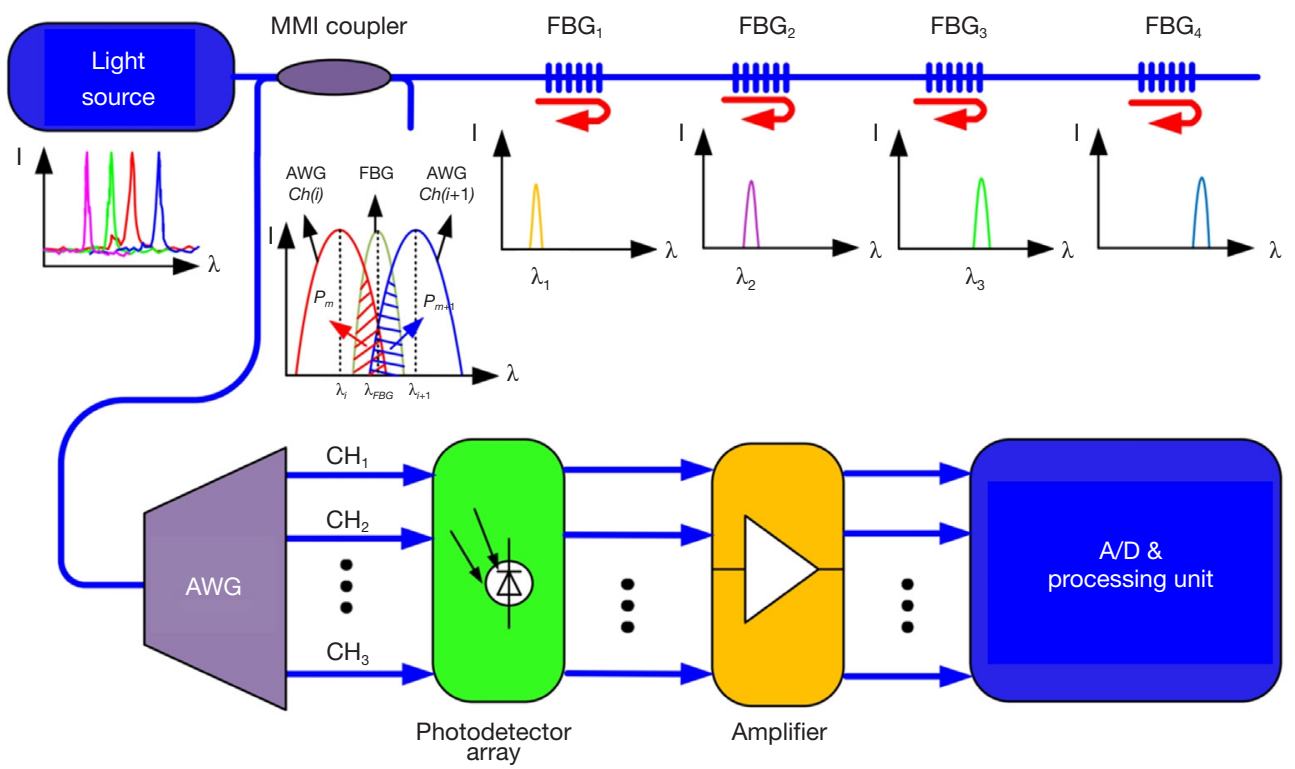

Figure 7 Structure of Bragg grating and experimental data of the FBG and $\pi$-FBG. (A) Transparent structure of Bragg grating. Adapted with permission from (49). Copyright 2014 AIP Publishing. (B) The spatial index variation of an FBG and a $\pi$-FBG. (C) The spectra of FBG and $\pi$-FBG. (B,C) are adapted with permission from (99). Copyright 2018 MDPI. (D) An FBG array. Adapted with permission from (50). Copyright 2017 OSA Publishing. FBG, fiber Bragg grating.

over a board bandwidth of $140 \mathrm{MHz}$ and a wide-field acceptance angle of $\pm 30^{\circ}$. The later and axial resolution was estimated to be 2.0 and $5.3 \mu \mathrm{m}$ with the ultrasound sensor, respectively. A phantom image is shown in Figure $6 B$.

It is convenient to integrate the microring resonators in form of an array (73), as shown in Figure 6C,6D. The $1 \times 5$ polymeric microring resonators array was coupled with single-mode polarization-maintaining fiber and multimode fiber for delivering and collecting laser radiation, respectively. The sensor was attached to a prism facet in a sealed endoscopic probe. The acquired NEP was $352 \mathrm{~Pa}$ over a $250 \mathrm{MHz}$ bandwidth. The resulting axial resolution was as precise as $4.5 \mu \mathrm{m}$. The volumetric imaging ability was shown in Figure 6 E. With the assistance of increased spatial resolution, the depth information less than $100 \mu \mathrm{m}$ was convenient to be reconstructed.

\section{Bragg grating sensors}

In 1996, Webb et al. (93) reported the first demonstration of FBGs for acoustic detection. Further works were extended to use arrayed waveguide gratings in FBG sensors (94), which exploited the capability of wavelength multiplexing. In this case, FBGs in line could probe the ultrasound simultaneously from multiple resonant wavelengths.

In order to detect acoustic pressure, Bragg grating sensors involve uniform FBGs, $\pi$-phase-shift FBGs ( $\pi$-FBGs) (95-97), and Bragg grating waveguides (BGWs) (48-50,98). Bragg grating is a transparent structure with a periodic variation of refractive index, as shown in Figure $7 A$. The Bragg's law can be defined as $\Lambda=\lambda / 2 \mathrm{n}_{\text {eff }}$, where $\Lambda$ is the spatial period of Bragg grating, $\lambda$ is the incident laser wavelength, and $n_{\text {eff }}$ is the effective refractive index of Bragg grating. When the law is fulfilled, the input optical fields are reflected by the structures of Bragg grating and 
the reflected optical fields interfere constructively at the resonant wavelength. As a result, a narrow passband and a corresponding stopband appear in the reflection and transmission spectra, respectively. During ultrasound detection, Bragg grating sensors are influenced by acoustic pressure, which could change the physical dimensions of sensing structures. Thus, the $\mathrm{n}_{\text {eff }}$ is modified and the resonant wavelength is shifted with high sensitivity. Ultrasonic signals can be obtained from monitoring intensity modulation at a fixed wavelength.

Various methods are proposed to improve pressure sensitivity. Rosenthal et al. (95) proposed a fiber-optic ultrasound sensor by recording reflective variations of a continuous-wave laser from a $\pi$-FBG. The spatial index difference of an FBG and a $\pi$-FBG is shown in Figure $7 B$. The $\pi$-FBG is considered as a special FP structure formed by two standard FBGs with a phase shift of $\pi$. Figure $7 C$ shows the simulated spectra of a normal FBG and a $\pi$-FBG. Compared with a normal FBG, the $\pi$-FBG has a broader frequency response and a higher sensitivity due to a sharper dip around resonant wavelength. Moreover, the propagating light is well confined in the phase-shift region owing to strong localization. The effective length of the sensing element could be shortened as much as possible to realize a broad bandwidth. For an FBG sensor with a $270-\mu \mathrm{m}$ length, the pressure sensitivity of $440 \mathrm{~Pa}$ and the bandwidth of $10 \mathrm{MHz}$ can be obtained (95). In order to increase sensitivity, the laser wavelength can be locked to an external cavity mode on the spectral slope of $\pi$-FBG (100). When the distributed-feedback diode (DFB) laser was in the self-injection-locked mode, the wavelength could be automatically pulled to the $\pi$-FBG resonant wavelength. The $\pi$-FBG in a self-injection-locked DFB obtained $35 \mathrm{~dB}$ higher sensitivity than that in a free-running DFB. It is convenient for Bragg grating sensors to be cascaded in a line array due to the reflective function mode. An FBG interrogator can be integrated on a hybrid silicon chip together with light sources, couplers, and photodetectors, as shown in Figure 7D. Benefitted from a silicon-on-insulator platform, the hybrid chip has a compact size of $5 \mathrm{~mm}$ by $3 \mathrm{~mm}$. Owing to the stability of the systematic package, high wavelength resolution is up to $1 \mathrm{pm}(50)$.

As is known, FP sensors and microring resonators cannot be miniaturized sufficiently and confine light to dimensions smaller than $50 \mu \mathrm{m}$. Bragg-grating-based ultrasound sensors have emerged as potential point-like broadband devices, which can overcome the drawbacks to physical dimensions of sensing elements and increase the resolution of imaging performance $(49,95)$. Rosenthal et al. (49) had first applied silicon-on-insulator technology to $\pi$-phase-shift waveguide Bragg grating $(\pi-W B G)$ for miniaturization in 2014. The cross-section area of $\pi$-WBG was $200 \mathrm{~nm}$ by $500 \mathrm{~nm}$, but the sensing length was $250 \mu \mathrm{m}$ with a bandwidth of $30 \mathrm{MHz}$. It should be noted that the large sensing length and narrow bandwidth impeded point-like detection. Shnaiderman et al. (98) proposed a detector with an actual sensing area of $220 \mathrm{~nm}$ by $500 \mathrm{~nm}$ based on silicon-oninsulator technology, which was termed silicon waveguideetalon detector (SWED). The size of this detector was four orders of magnitude smaller than the smallest polymer microring detectors (98). The point-like SWED also enabled an ultrawide bandwidth of $230 \mathrm{MHz}$ at $-6 \mathrm{~dB}$, which paved the way for high dense ultrasonic arrays on a silicon chip and high-resolution photoacoustic microscopy.

\section{Sensors based on SPR}

Optical ultrasound sensors based on SPR have been widely used in photoacoustic microscopy $(52,101)$. The surface plasmon is optically coupled at a metal-dielectric interface. The refractive index of the coupling resonance is perturbed by photoacoustic signals. The PA signals can be measured by detecting light reflectivity variations (52). The SPR sensors generally employ an optical prism with thin metallic films. Laser pulses with p-polarization illuminate the prism to generate attenuated total reflection and excite surface plasmon polariton in metallic layers. The occurrence of SPR and enhancement of coupling between laser energy and surface plasmon polariton in the evanescent field are achieved when the wave vectors of incident pulses and surface plasmon polariton match with each other. In ultrasound detection, photoacoustic signals are detected by measuring amplitude and phase variations of probe pulses, which are changed by ultrasound-induced and ultrasoundmodulated SPR in metallic layers.

The bandwidth of SPR sensors is determined by physical dimensions of the evanescent field, which is in the level of acoustic wavelength and theoretically corresponds to the bandwidth of GHz (102). However, the actual bandwidth fails to reach the $\mathrm{GHz}$ level. The researchers aim to increase the actual bandwidth of SPR sensors and improve axial resolutions for volumetric imaging. Wang et al. (103) reported on SPR-based photoacoustic microscopy with ultra-flat frequency response from 0.68 to $126 \mathrm{MHz}$. Linear frequency response was confirmed from $5.2 \mathrm{kPa}$ to 2.1 $\mathrm{MPa}$ with an NEP of $3.3 \mathrm{kPa}$. For ex vivo PAI, a single melanoma cell was reconstructed in a three-dimension 

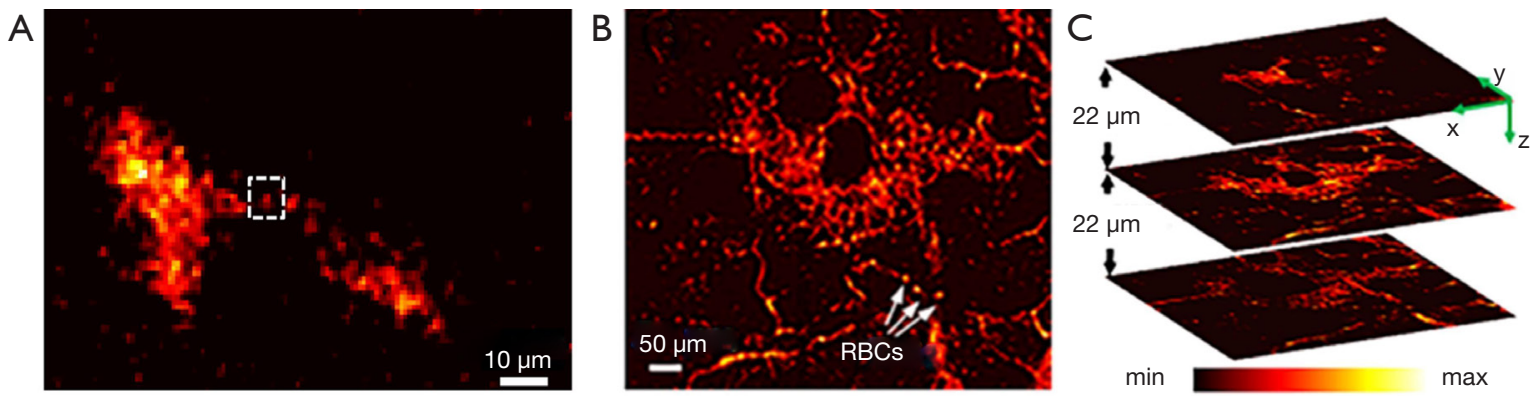

Figure 8 Experimental images obtained by SPR sensors. (A) High-resolution PAI based on SPR sensors. Adapted with permission from (103). Copyright 2015 AIP Publishing. (B) In vivo vasculature images of capillary bed of a mouse ear. (C) Depth-resolved capillary bed images. (B,C) are adapted with permission from (52). Copyright 2019 ACS. SPR, surface plasmon resonance; PAI, photoacoustic imaging.

model with lateral and axial resolutions of 2.0 and $8.4 \mu \mathrm{m}$, respectively, as shown in Figure $8 \mathrm{~A}$. The excellent bandwidth and flat frequency response led to comparable resolutions in lateral and depth information. Yang et al. and Song et al. $(52,104-106)$ from the same group demonstrated a series of works on SPR sensors and biomedical applications. Various approaches have been tried to increase the bandwidth and sensitivity of SPR sensors, including the use of graphene instead of metallic films, inserting an acoustic cavity, and polarization-differential detection. Among these approaches, polarization-differential detection (52) showed the best performance of $173-\mathrm{MHz}$ bandwidth and lateral and axial resolutions of 4.5 and $7.6 \mu \mathrm{m}$ in Figure 8B, respectively. As shown in Figure $8 C$, in vivo threedimensional PAI was demonstrated in a mouse ear with a $22-\mu \mathrm{m}$ isometric distance.

\section{Non-resonance-based optical ultrasound sensors and applications}

The non-resonance-based optical ultrasound sensors obtain the images of biological tissues by measuring the beam deflection, amplitude modulation and phase change of the detection light. Non-resonance-based optical ultrasound sensors consist of Mach-Zehnder interferometer (MZI), Michelson interferometer (MI), optical camera and probe beam deflection techniques (PBDT).

\section{Sensors based on MZIs}

In 1977, Bucaro et al. (107) first reported on an MZIbased fiber acoustic sensor with a frequency response of $40-400 \mathrm{kHz}$ and an NEP of $0.1 \mathrm{~Pa}$. A typical MZI consists of two fiber couplers that serve as reference and sensing paths of an interferometer. Laser pulses from the reference path interfere with interrogation pulses reflecting from the sensing path that contains pressure-encoded information. The modulation of interferometric signals is utilized to quantitatively reflect surface displacement, which is scalable with the amplitude of acoustic waves. The interferometric sensor can precisely obtain the information of ultrasound by analyzing the interference spectrum, which is induced by the phase difference between the optical paths. Figure 9 presents a setup of the Mach-Zehnder interferometric principle.

MZI-based sensors utilize optical fibers as sensing elements or directly collect spatially scattering light with high efficiency to detect ultrasound signals. In the condition of optical fibers as sensing elements, the fibers in the detecting path become deformed due to the elasticoptic effect under external acoustic pressure. However, the sensing element of interferometer is fiber length, which ultimately limits the performance of ultrasound sensing. For instance, it could detect high-frequency signals up to tens of $\mathrm{kHz}$. In the detection, only a small section of optical fibers is deformed by pressure-induced strain while the entire optical fibers are still insensitive. As for directly collecting spatial light, non-contact detection of acoustic waves from rough surfaces is realizable without sensing elements. Non-contact PAI based on all-fiber MZI is free from physical contacts or impedance matching media (108). Different from the conventional configuration, the use of lensed fibers instead of free-space focusing optics brought the non-contact method to medical applications. Previous photoacoustic microscopy employs high-frequency physical ultrasound transducers or resonance-based sensors to measure the acoustic pressure (109-111). The acoustic coupling media are needed to decrease insertion loss induced by mismatches of targets and sensors, limiting various applications of in vivo imaging. However, MI- and 


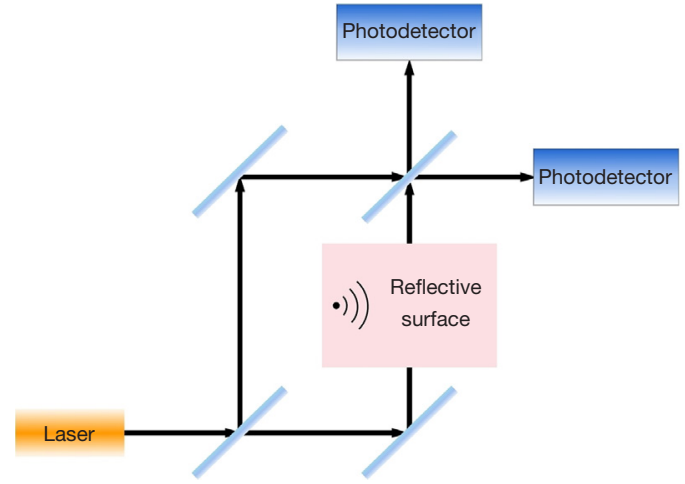

Figure 9 Setup of the Mach-Zehnder interferometric sensor principle.
MZI-based ultrasound sensors acquire pressure information by probing the photoacoustic-signals-induced phase or intensity changes of interrogation light, making the noncontact approach come true. A $3 \times 3$ coupler-based MachZehnder interferometric system was demonstrated for noncontact imaging in Figure 10A-10C (112). The impact of the rough tissue surface was eliminated by measuring the intensity change instead of phase change, fulfilling the goal of non-contact imaging in real time. The microvasculature of the mouse ear was clearly visualized with an $11-\mu \mathrm{m}$ lateral resolution in the measurement. The use of $3 \times 3$ optical couplers helped to obtain phase information of the $\mathrm{MZ}$ interferometer. Besides, a high-pass filter was applied
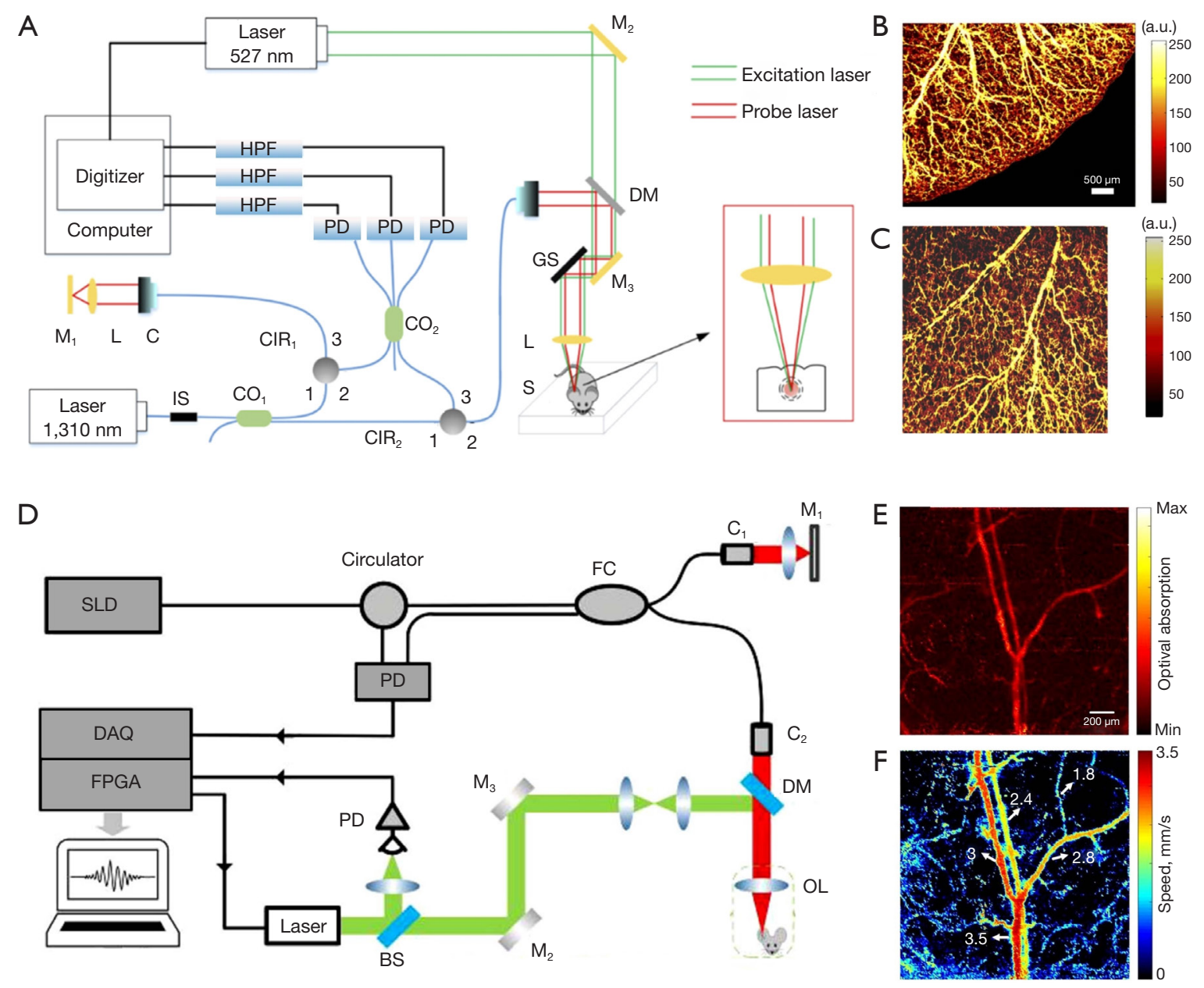

Figure 10 Schematic illustrations of Mach-Zehnder interferometric systems and experimental images. (A) An MZI-based photoacoustic microscopy. The maximal amplitude projection images of two different regions of a mouse ear are plotted in (B,C). (D) An MI-based ultrasound detection for blood flow imaging. (E) Microvascular structure of a mouse ear. (F) Blood flow of the blood vessel. (A-C) are adapted with permission from (112). Copyright 2020 OSA Publishing. (D-F) are adapted with permission from (113). Copyright 2018 OSA Publishing. MZI, Mach-Zehnder interferometer; MI, Michelson interferometer. 
to reduce complex interference and transmission of signals at high frequency. Hence, MZI-based sensors are suitable for high-speed imaging without phase stabilization. Apart from MZI-based sensors, MI-based sensors are capable to collect scattered light with high efficiency and realize noncontact imaging in the dermatology.

\section{Sensors based on MIs}

In order to simplify the MZI structure, fiber optic acoustic sensors based on MI were designed with one coupler (114). At the end of the coupler, high-reflecting mirrors were attached with sensing and reference paths. The sensor based on MI utilized a low-coherence laser source with a lateral resolution of $30 \mu \mathrm{m}$ (115). It can detect backscattering light from different depths via coherence gating, avoiding the ambiguous interpretation of the detected signals.

It is a common problem for MI sensors that ambient vibrations cause an additional deforming, leading to a fluctuation of the systematic sensitivity. It is required to lock the system at the highest sensitivity for mitigation of perturbation, which slows down the imaging speed in return. Multiport optical couplers were employed to overcome this challenge in novel quadrature detection, such as $3 \times 3$ couplers $(116,117)$. This kind of couplers has an intrinsic phase difference of $120^{\circ}$. The final interferometric signal could be extracted with $3 \times 3$ couplers to overcome the fluctuating sensitivity problem. The homodyne detection is simple and has no demand for a feedback loop, which attracts attention in high-speed applications.

MI-based ultrasound sensors are also suitable for noncontact photoacoustic microscopy. As shown in Figure 10D, all-optical photoacoustic microscopy was based on an MI to map the images of blood flow and velocity (113). The microvascular structure and blood flow of a mouse ear were shown in Figure 10E,10F. The lateral and axial resolutions were measured to be 11 and $19.7 \mu \mathrm{m}$, respectively. The velocity value of the vascular trunk was $3.5 \mathrm{~mm} / \mathrm{s}$ while the velocity values of branches were 3, 2.8, 2.4 and $1.8 \mathrm{~mm} / \mathrm{s}$, respectively. The information of blood flow represents the status of the metabolism, which is used to evaluate angiocardiopathy and atherosclerosis.

\section{Optical camera for ultrasound detection}

Optical cameras have been used to measure acoustic field by using Schlieren technique (118) and phase contrast method $(119,120)$. The Schlieren technique can visualize ultrasound signal in transparent media, which is realized by deflection of light in gradient field of the refractive index (121). In the ultrasonic detection, a simple phase contrast optical signal processing element is employed. Hence, it is termed optical phase contrast method (120). Charge coupled devices (CCD) are widely used in commercial and scientific fields. The principles of ultrasonic detection by CCD are based on the conversion of ultrasound-induced phase shift into optical intensity mapping. In both conditions of Schlieren and phase contrast method, Fourier lenses are used to form a confocal plane. The spatial Fourier spectrum of a target on the confocal plane represents the distribution of optical intensity. The difference between two methods exists in spatial filtering optics. Schlieren technique employs an aperture diaphragm while phase contrast method uses a phase plate (122).

Typical photoacoustic microscopy based on CCD has been demonstrated to image blood vessels of a mouse by Nuster et al. (119). In a phantom imaging experiment, the estimated axial and lateral resolution was around 73 and $80 \mu \mathrm{m}$, respectively. In vivo imaging of the left leg of a mouse, one hundred projection images could be recorded for $3 \mathrm{D}$ reconstruction in $3 \mathrm{~min}$. As a result, blood vessels were demonstrated with a lateral resolution of less than $100 \mu \mathrm{m}$ in Figure 11A,11B. It should be noted that the sensitivity of optical phase contrast detection was $5.1 \mathrm{kPa}$ and the detectable frequency range was cut off from 1.1 to $23 \mathrm{MHz}$. The advantages of CCD as an ultrasound sensor are capabilities of parallel detection, single-shot recording, and real-time $2 \mathrm{D}$ imaging, but the limited bandwidth and sensitivity hinder the high resolution and SNR.

\section{Sensors based on PBDT}

Ultrasonic signals can be measured by PBDT (123-125). During optical ultrasound detection, a probe beam passes through a transparent medium. At the same time, the refractive index of the propagating medium is proportionally affected by pressure gradients of ultrasonic signals. The beam deflection occurs at the intersection of the probe beam and acoustic wavefront. Finally, the deflection angle is measured by a position-sensitive photodiode connected to a differential amplifier.

It should be noted that the resolution of PBDT mainly depends on the wavelength and diameter beam of the probe beam, rather than the performance of ultrasound sensors. The theoretical lateral resolution is calculated by $0.5 \lambda / \mathrm{NA}$ and the axial resolution is primarily defined by the depth of field which can be expressed as $\lambda \mathrm{n} / \mathrm{NA}^{2}$, where $\lambda$ is the wavelength of the probe beam, $\mathrm{n}$ is the refractive index of transmitting media, and NA is the numerical aperture of 

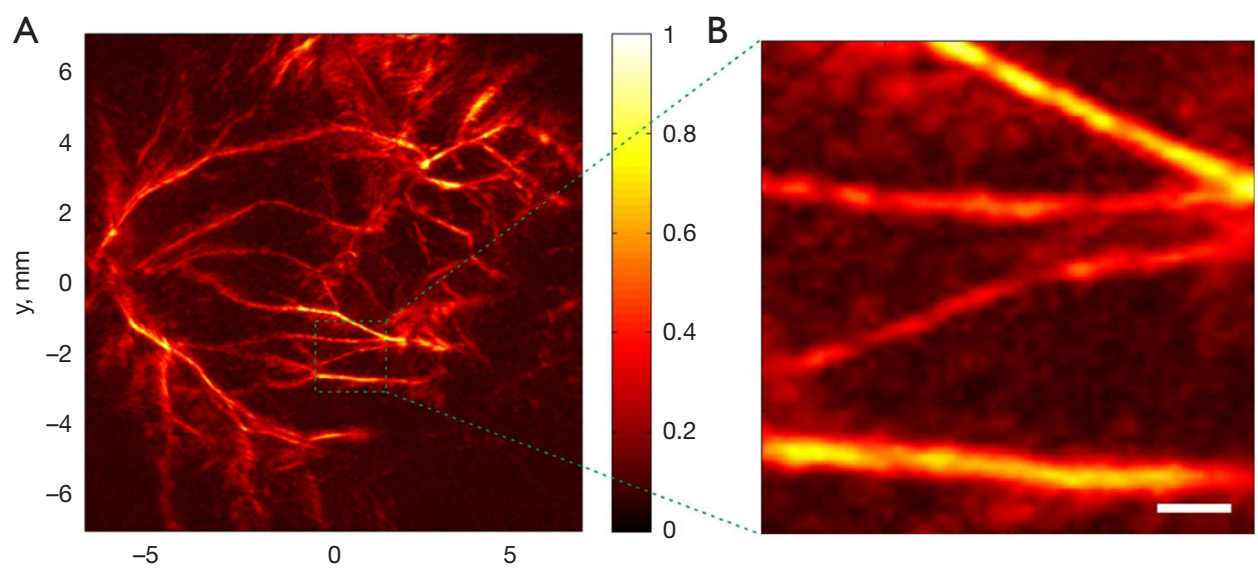

C

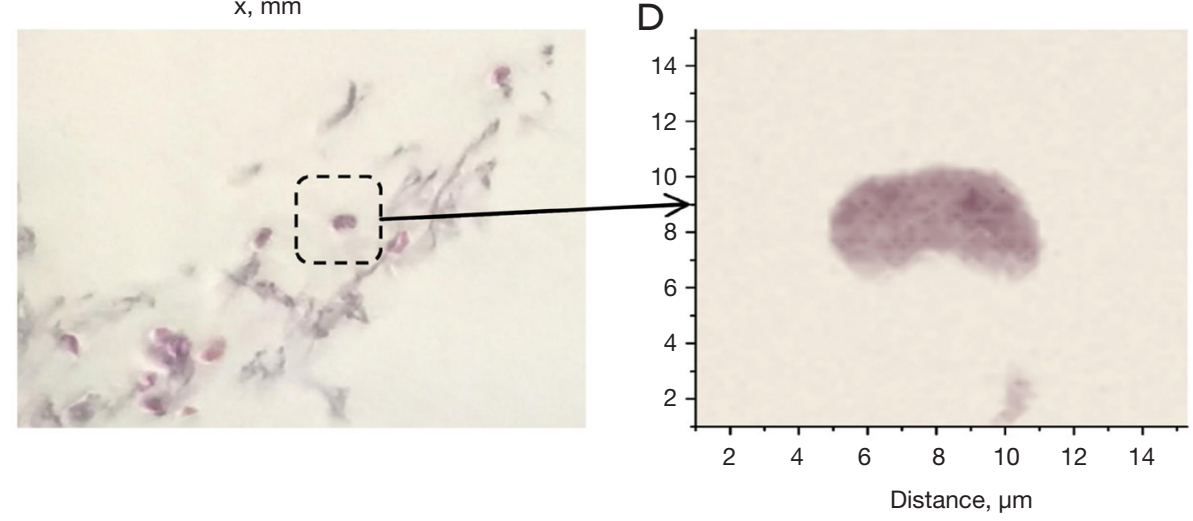

Figure 11 Experimental images of photoacoustic imaging based on CCD and PBDT detection. (A) Photoacoustic MAP images from a top view of the left hind leg of a mouse. (B) A zoom of small blood vessels in the dashed box in (A). (C) Optical image of a sample with tissue debris and cluster of lysed red blood cells. (D) Photoacoustic image of the single erythrocyte. (A,B) are adapted with permission from (119). Copyright 2014 OSA Publishing. (C,D) are adapted with permission from (123). Copyright 2016 Elsevier. CCD, charge coupled devices; PBDT, probe beam deflection techniques; MAP, maximum amplitude projection.

the probe beam. Compared with conventional transducers, PBDT possesses high sensitivity, ultrawide bandwidth, as well as avoiding the separation of optical and acoustic paths thus achieving the undistorted coupling of acoustic waves to the detector (123). In order to improve the resolution of PBDT, combining PBDT with the best high numerical aperture objectives or integrating PBDT with other optical imaging techniques that are used in an optical microscope are both promising methods (124).

Maswadi et al. (123) demonstrated that the in vitro experiments can be implemented by PAI of PBDT detection. Fixed biological samples of cardiac tissues and single erythrocyte were mechanically scanned with high resolution, as depicted in Figure 11C,11D. The estimated axial and lateral resolution were 0.7 and $0.5 \mu \mathrm{m}$, respectively. The ability to detect single cells is highly desirable for molecular cell biology (12). The bandwidth of this PBDT system was nearly $17 \mathrm{MHz}$, determined by the division of sound speed and beam diameter. The sensitivity of PBDT detection was estimated to be $11.4 \mathrm{~Pa}$, which was calibrated by a hydrophone with certain sensitivity. In PBDT, excitation laser pulses are tightly focused, leading to a small focal depth. The path of the probe beam needs to be closely located at the tissue surface. Hence, PBDT with high sensitivity and ultrawide bandwidth is suitable for photoacoustic microscopy with small active sensing areas.

\section{Perspectives}

Optical ultrasound detection has emerged and matured as an efficient technique for visualizing distributions in biological imaging. Traditional piezoelectric and micromachined 
Table 1 Summary of characteristic parameters of optical ultrasound sensors

\begin{tabular}{|c|c|c|c|c|c|c|c|}
\hline Category & $\begin{array}{c}\mathrm{NEP} \\
\left(\mathrm{mPa} / \mathrm{Hz}^{1 / 2}\right)\end{array}$ & $\begin{array}{l}\text { Central frequency } \\
(\mathrm{MHz})\end{array}$ & $\begin{array}{l}\text { Bandwidth at } \\
-3 \mathrm{~dB}(\mathrm{MHz})\end{array}$ & Q factor & $\begin{array}{l}\text { Axial/lateral } \\
\text { resolution }(\mu \mathrm{m})\end{array}$ & $\begin{array}{l}\text { Acceptant } \\
\text { angle }\end{array}$ & Ref. \\
\hline \multicolumn{8}{|l|}{ Resonance } \\
\hline $\mathrm{FP}$ & 2.1 & 3.5 & 20 & $>10^{5}$ & $36 /-$ & 90 & (46) \\
\hline$\pi-W B G$ & - & 15 & 20 & - & - & 26.6 & (49) \\
\hline BGW & 9 & 37 & 103 & $2.2 \times 10^{4}$ & $0.65 / 0.65$ & 90 & (98) \\
\hline BGW & 710 & - & 60 & - & - & - & (48) \\
\hline Microring & 0.038 & 84 & 166 & $1.4 \times 10^{5}$ & $3.57 /-$ & - & (125) \\
\hline SPR & 294 & $0.7-126$ & 126 & - & $8.4 / 2.0$ & - & (102) \\
\hline SPR & 36.3 & - & $173(-6 \mathrm{~dB})$ & - & $7.6 / 4.5$ & - & (52) \\
\hline \multicolumn{8}{|c|}{ Non-resonance } \\
\hline CCD & $5.3(\mathrm{kPa})$ & $1.1-23$ & - & - & $73 / 80$ & - & (118) \\
\hline PBDT & $11.4(\mathrm{~Pa})$ & 17 & - & - & $1.0 / 0.5$ & - & (124) \\
\hline MZI & $0.1(\mathrm{~Pa})$ & $0.04-0.4$ & - & - & - & - & (106) \\
\hline
\end{tabular}

NEP, noise equivalent pressure; Q, quality; FP, Fabry-Perot; FBG, fiber Bragg grating; WBG, waveguide Bragg grating; BGW, Bragg grating waveguide; SPR, surface plasmon resonance; CCD, charge coupled devices; PBDT, probe beam deflection techniques; MZI, MachZehnder interferometer; MI, Michelson interferometer.

ultrasound transducers own high sensitivity, but fail to decrease the size of piezoelectric transducers while keeping broad bandwidth over tens of MHz. However, optical ultrasound sensors can overcome the limitations, setting new performance standards for all-optical PAI. A summary of basic characteristics of optical ultrasound sensors is listed in Table 1, including NEP, bandwidth, resolutions, central frequency, $\mathrm{Q}$ factor and acceptant angle. The sensitivity is commonly quantified by the NEP, in which a small NEP refers to a better sensitivity.

Resonance-based ultrasound sensors, mainly including FP, Bragg grating, and microring sensors, have the capabilities to reach high sensitivity where the NEP is less than $10 \mathrm{mPa} / \mathrm{Hz}^{1 / 2}$. Sufficient SNR benefits from the precise NEP. These sensors are compatible with optical fibers and silicon-on-insulator platforms, which realize the compact size and electromagnetic interference immunity. In addition, FP, Bragg grating, and microring sensors have shown the abilities in high-dense arrays for parallel detection, decreasing the acquisition time of $2 \mathrm{D}$ and $3 \mathrm{D}$ imaging. Spatial resolution at the level of micrometers could be achieved in Bragg grating, microring and SPR sensors with bandwidths broader than $100 \mathrm{MHz}$. Ex vivo and in vivo experiments have been carried out with these sensors in photoacoustic microscopy. For instance, Shnaiderman et al. (98) demonstrated super-resolution 
imaging by Bragg grating sensors, where the resolution was 50 times smaller than acoustic wavelength. Li et al. (126) recorded cortical vessels of live mice for 28 days using photoacoustic microscopy based on microring sensors. It is desirable that FP sensors are utilized in photoacoustic endoscopy with satisfactory moderate resolution. The FP sensors are attached to distal ends of optical fibers with small footprints that are favorable to minimally invasive interventions, such as fetal surgery and endometrial diseases. Tunable continuous-wave lasers with narrow linewidth are used as the interrogation light combined with resonancebased optical ultrasound sensors, but the high cost of lasers limits widespread applications of optical ultrasound detection. However, there still exist inherent drawbacks of resonance-based ultrasound sensors. For FP and Bragg grating sensors, tunable narrow line-width continuous wave laser is required, which is expensive. For microring sensors, the fabrication process is difficult and the life span is short in biological tissues. It is not suitable for SPR sensors to be used in arrayed detection and parallel detection.

Non-resonance-based sensors based on MI, MZI, CCD and PBDT have specific advantages and drawbacks. MI and MZI based sensors are suitable for non-contact PAI, especially avoiding the second damage to tissues with ulcerations in the detection. The CCD-based sensors have access to parallel detection and single-shot imaging. However, further development is limited by the insufficient sensitivity and narrow bandwidth. Sensors based on PBDT have high sensitivity and resolution which depend on the wavelength and diameter of the probe beam, but they are restricted by long scanning time and short effective scanning region. Moreover, there are inadequate sensitivity and narrow bandwidth for MZI, MI and CCD. For PBDT, the scanning time is long and the detection range is limited. Non-resonance-based sensors still need constant development to overcome the weaknesses. The merits and drawbacks of various optical ultrasound sensors are summarized in Table 2.

PAI based on optical ultrasound sensors can provide structural, functional and molecular information of biological tissues $(24,127)$. Owing to the energy delivery mechanism, PAI possess deep imaging capability that could penetrate deep tissues successfully. However, the resolution of PAI decreases with the increase of the penetration depth (128). In order to enhance the resolution of PAI in achieving deep tissues imaging, the integration of PAI and other optical imaging technique is of great significance, such as combing
PAI with optical coherence tomography (OCT). OCT, an imaging technology with high resolution, is extensively used in noninvasive imaging (129). Besides, it focuses on the detection of backscattered photons of targeted tissues excited by a low-coherence light source. Compared with PAI, OCT could achieve tissues imaging with the resolution of several to tens of microns (130). Whereas, OCT suffers from shallow tissue penetration of single millimeters degraded by optical scattering and also fails to provide complementary information. The modality of PAI includes optical and acoustic paths for signal processing, which makes it convenient to integrate with OCT $(129,131)$. The dual-modal combination of PAI and OCT is promising to overcome specific limitations of a single modality $(132,133)$. For instance, Li et al. (134) set up the dual-modality microscope, where PAI combined with OCT achieved comprehensive information about biological tissues. They obtained three-dimensional imaging of microvasculature in vivo and proved the images with micrometer-order resolution. Jiao et al. (135) integrated non-invasive PAI with spectral-domain OCT, obtaining the microanatomy and microvasculature of the rat retina with high resolution in vivo.

Besides optical ultrasound sensors, the performances of laser sources are significant for PAI. There are two potential trends for promoting the development of laser sources in PAI, including low-cost light emitting diodes and high-performance laser sources (136-140). Recently, optical frequency combs are combined with ultrasound sensors to enable precise photoacoustic spectroscopy (141-145). Optical frequency combs are specific light sources that provide coherent broad spectra featured with evenly spaced optical frequencies (146). They have been used in various applications of time standards, metrology and spectroscopy. Broadband spectra are promptly excited in the resonant cavity and then recorded by sequential snapshots of a single optical frequency comb. This novel method makes the spectrum-resolved PAI feasible for realtime monitoring and high-precision detection. Moreover, mechanical scanning and wavelength tuning could be replaced in the dual-comb photoacoustic modality owing to the coherence of two combs (145).

The properties of optical ultrasound sensors will be improved with the assistance of silicon-on-insulator technology, including high sensitivity, broad bandwidth, point-like aperture and high-density array. Combined with cheap light emitting diodes, PAI based on optical ultrasound sensors is supposed to be commercialized in 
Table 2 The advantages and disadvantages of optical ultrasound sensors

\begin{tabular}{|c|c|c|}
\hline Category & Advantages & Disadvantages \\
\hline \multicolumn{3}{|l|}{ Resonance } \\
\hline FP & $\begin{array}{l}\text { - High sensitivity } \\
\text { - Broad bandwidth } \\
\text { - Wide-angle detection } \\
\text { - Small footprint } \\
\text { - Simple and inexpensive sensor fabrication } \\
\text { - Safety for endoscopic use } \\
\text { - Immune to radiofrequency interference }\end{array}$ & $\begin{array}{l}\text { Require tunable narrow line-width continuous } \\
\text { wave laser with high cost }\end{array}$ \\
\hline Microring & $\begin{array}{l}\text { - High acoustic detection sensitivity } \\
\text { - Ultrabroad bandwidth } \\
\text { - Wide angular response } \\
\text { - Compact configuration for high-density arrays } \\
\text { immune to radiofrequency interference }\end{array}$ & $\begin{array}{l}\text { - Difficult fabrication process } \\
\text { - Short life span in biological tissues }\end{array}$ \\
\hline Bragg grating & $\begin{array}{l}\text { - Compact configuration for high-density arrays } \\
\text { - High sensitivity } \\
\text { - Broad bandwidth } \\
\text { - Immune to radiofrequency interference }\end{array}$ & $\begin{array}{l}\text { Require tunable narrow line-width continuous } \\
\text { wave laser with high cost }\end{array}$ \\
\hline SPR & - Broad bandwidth & $\begin{array}{l}\text { - Not suitable for arrayed detection and parallel } \\
\text { detection } \\
\text { - Low imaging speed }\end{array}$ \\
\hline \multicolumn{3}{|l|}{ Non-resonance } \\
\hline CCD & $\begin{array}{l}\text { - Parallel detection } \\
\text { - Fast imaging } \\
\text { - Single shot imaging }\end{array}$ & $\begin{array}{l}\text { - Inadequate sensitivity } \\
\text { - Narrow bandwidth }\end{array}$ \\
\hline PBDT & $\begin{array}{l}\text { - High sensitivity } \\
\text { - Broad bandwidth }\end{array}$ & $\begin{array}{l}\text { - Long scanning time } \\
\text { - Limited scanning range }\end{array}$ \\
\hline
\end{tabular}

FP, Fabry-Perot; SPR, surface plasmon resonance; MZI, Mach-Zehnder interferometer; MI, Michelson interferometer; CCD, charge coupled devices; PBDT, probe beam deflection techniques.

biomedical applications. Meanwhile, high-performance light sources of optical frequency combs will pursue optimized performance in PAI.

\section{Conclusions}

In this work, we review optical ultrasound sensors based on resonance and non-resonance principles, including FP, 
microring, Bragg grating and SPR sensors, as well as MI, MZI, CCD and PBDT sensors. The characteristics and development of optical ultrasound sensors are presented in detail. Optical ultrasound sensors provide a bright prospect in biomedical fields, including photoacoustic endoscopy, microscopy and non-contact imaging. The merits and drawbacks of resonance- and non-resonance-based sensors are discussed in terms of high sensitivity, high-density array, non-contact sensing and compatibility with silicon-oninsulator platforms. For resonance-based sensors, compact FP sensors in fiber optics are suitable for photoacoustic endoscopy. Microring and Bragg grating sensors are employed in the microscopy owing to the high bandwidth and resolution. For non-resonance-based sensors, MZI and MI have high efficiency to collect scattered light, enabling non-contact imaging. CCD are used in fast PAI due to parallel detection. Moreover, these sensors are able to integrate with OCT to achieve high resolution at surface and adequate resolution in deep tissues. Optical ultrasound sensors with high sensitivity, broad bandwidth and parallel detection are expected to accelerate the pace of innovations in biomedical imaging.

\section{Acknowledgments}

Funding: The work was supported by the National Key Research and Development Program of China (2018YFB2003200), National Natural Science Foundation of China [62071016], Beijing Natural Science Foundation [4202044], Open Fund of IPOC (BUPT), and Fundamental Research Funds for the Central Universities.

\section{Footnote}

Reporting Checklist: The authors have completed the Narrative Review reporting checklist. Available at https:// dx.doi.org/10.21037/qims-21-605

Conflicts of Interest: All authors have completed the ICMJE uniform disclosure form (available at https://dx.doi. org/10.21037/qims-21-605). All authors report that the work was partly supported by the National Key Research and Development Program of China (2018YFB2003200), National Natural Science Foundation of China (62071016), Beijing Natural Science Foundation (4202044), Open Fund of IPOC (BUPT), and Fundamental Research Funds for the Central Universities. The authors have no other conflicts of interest to declare.
Ethical Statement: The authors are accountable for all aspects of the work in ensuring that questions related to the accuracy or integrity of any part of the work are appropriately investigated and resolved.

Open Access Statement: This is an Open Access article distributed in accordance with the Creative Commons Attribution-NonCommercial-NoDerivs 4.0 International License (CC BY-NC-ND 4.0), which permits the noncommercial replication and distribution of the article with the strict proviso that no changes or edits are made and the original work is properly cited (including links to both the formal publication through the relevant DOI and the license). See: https://creativecommons.org/licenses/by-nc-nd/4.0/.

\section{References}

1. Lin L, Hu P, Shi J, Appleton CM, Maslov K, Li L, Zhang R, Wang LV. Single-breath-hold photoacoustic computed tomography of the breast. Nat Commun 2018;9:2352.

2. Kruger RA, Kuzmiak CM, Lam RB, Reinecke DR, Del Rio SP, Steed D. Dedicated 3D photoacoustic breast imaging. Med Phys 2013;40:113301.

3. Lan L, Xia Y, Li R, Liu K, Mai J, Medley JA, Obeng-Gyasi S, Han LK, Wang P, Cheng JX. A fiber optoacoustic guide with augmented reality for precision breast-conserving surgery. Light Sci Appl 2018;7:2.

4. Zhu Q, Huang M, Chen N, Zarfos K, Jagjivan B, Kane M, Hedge P, Kurtzman SH. Ultrasound-guided optical tomographic imaging of malignant and benign breast lesions: initial clinical results of 19 cases. Neoplasia 2003;5:379-88.

5. Ma H, Cheng Z, Wang Z, Zhang W, Yang S. Switchable optical and acoustic resolution photoacoustic dermoscope dedicated into in vivo biopsy-like of human skin. Appl Phys Lett 2020;116:073703.

6. Nitkunanantharajah S, Zahnd G, Olivo M, Navab N, Mohajerani P, Ntziachristos V. Skin Surface Detection in 3D Optoacoustic Mesoscopy Based on Dynamic Programming. IEEE Trans Med Imaging 2020;39:458-67.

7. Dahlstrand U, Sheikh R, Berggren J, Hult J, Albinsson J, Cinthio M, Malmsjö M. Spectral Signatures in the Different Layers of the Human Eyelid by Photoacoustic Imaging. Lasers Surg Med 2020;52:341-6.

8. Schwarz M, Soliman D, Omar M, Buehler A, Ovsepian SV, Aguirre J, Ntziachristos V. Optoacoustic Dermoscopy of the Human Skin: Tuning Excitation Energy for Optimal Detection Bandwidth With Fast and Deep Imaging in 
vivo. IEEE Trans Med Imaging 2017;36:1287-96.

9. Wang Y, Meng R, Song H, Liu G, Hua Y, Cui D, Zheng L, Feng W, Liebeskind DS, Fisher M, Ji X. Remote Ischemic Conditioning May Improve Outcomes of Patients With Cerebral Small-Vessel Disease. Stroke 2017;48:3064-72.

10. Chen J, Liu L, Zhang H, Geng X, Jiao L, Li G, Coutinho JM, Ding Y, Liebeskind DS, Ji X. Endovascular Hypothermia in Acute Ischemic Stroke: Pilot Study of Selective Intra-Arterial Cold Saline Infusion. Stroke 2016;47:1933-5.

11. van der Sanden B, Hugon O, Inglebert M, Jacquin O, Lacot E. Vascular bifurcation mapping with photoacoustic microscopy. Biomed Opt Express 2020;11:1298-305.

12. Wang LV, Yao J. A practical guide to photoacoustic tomography in the life sciences. Nat Methods 2016;13:627-38.

13. Hariri A, Fatima A, Mohammadian N, Mahmoodkalayeh S, Ansari MA, Bely N, Avanaki MRN. Development of lowcost photoacoustic imaging systems using very low-energy pulsed laser diodes. J Biomed Opt 2017;22:75001.

14. Allen TJ, Beard PC. High power visible light emitting diodes as pulsed excitation sources for biomedical photoacoustics. Biomed Opt Express 2016;7:1260-70.

15. Agrawal S, Kuniyil Ajith Singh M, Johnstonbaugh K, C Han D, R Pameijer C, Kothapalli SR. Photoacoustic Imaging of Human Vasculature Using LED versus Laser Illumination: A Comparison Study on Tissue Phantoms and In Vivo Humans. Sensors (Basel) 2021;21:424.

16. Fatima A, Kratkiewicz K, Manwar R, Zafar M, Zhang R, Huang B, Dadashzadeh N, Xia J, Avanaki KM. Review of cost reduction methods in photoacoustic computed tomography. Photoacoustics 2019;15:100137.

17. Hunt JW, Arditi M, Foster FS. Ultrasound transducers for pulse-echo medical imaging. IEEE Trans Biomed Eng 1983;30:453-81.

18. Zhou Q, Lau S, Wu D, Shung KK. Piezoelectric films for high frequency ultrasonic transducers in biomedical applications. Prog Mater Sci 2011;56:139-74.

19. Ma J, Guo S, Wu D, Geng X, Jiang X. Design, fabrication, and characterization of a single-aperture $1.5-\mathrm{MHz} / 3-\mathrm{MHz}$ dual-frequency HIFU transducer. IEEE Trans Ultrason Ferroelectr Freq Control 2013;60:1519-29.

20. Oralkan O, Ergun AS, Johnson JA, Karaman M, Demirci U, Kaviani K, Lee TH, Khuri-Yakub BT. Capacitive micromachined ultrasonic transducers: next-generation arrays for acoustic imaging? IEEE Trans Ultrason Ferroelectr Freq Control 2002;49:1596-610.

21. Akasheh F, Myers T, Fraser JD, Bose S, Bandyopadhyay A.
Development of piezoelectric micromachined ultrasonic transducers. Sensor Actuat a-Phys 2004;111:275-87.

22. Qiu Y, Gigliotti JV, Wallace M, Griggio F, Demore CE, Cochran S, Trolier-McKinstry S. Piezoelectric micromachined ultrasound transducer (PMUT) arrays for integrated sensing, actuation and imaging. Sensors (Basel) 2015;15:8020-41.

23. Khuri-Yakub BT, Oralkan O. Capacitive micromachined ultrasonic transducers for medical imaging and therapy. J Micromech Microeng 2011;21:54004-14.

24. Manwar R, Kratkiewicz K, Avanaki K. Overview of Ultrasound Detection Technologies for Photoacoustic Imaging. Micromachines (Basel) 2020;11:692.

25. Caliano G, Carotenuto R, Cianci E, Foglietti V, Caronti A, Iula A, Pappalardo M. Design, fabrication and characterization of a capacitive micromachined ultrasonic probe for medical imaging. IEEE Trans Ultrason Ferroelectr Freq Control 2005;52:2259-69.

26. Zamora I, Ledesma E, Uranga A, Barniol N. Miniaturized 0.13- $\mu \mathrm{m}$ CMOS Front-End Analog for AlN PMUT Arrays. Sensors (Basel) 2020;20:1205.

27. Chee R, Sampaleanu A, Rishi D, Zemp R. Top orthogonal to bottom electrode (TOBE) 2-D CMUT arrays for 3-D photoacoustic imaging. IEEE Trans Ultrason Ferroelectr Freq Control 2014;61:1393-5.

28. Vallet M, Varray F, Boutet J, Dinten JM, Caliano G, Savoia AS, Vray D. Quantitative comparison of PZT and CMUT probes for photoacoustic imaging: Experimental validation. Photoacoustics 2017;8:48-58.

29. Zhu X, Huang Z, Wang G, Li W, Zou D, Li C. Ultrasonic detection based on polarization-dependent optical reflection. Opt Lett 2017;42:439-41.

30. Wissmeyer G, Pleitez MA, Rosenthal A, Ntziachristos V. Looking at sound: optoacoustics with all-optical ultrasound detection. Light Sci Appl 2018;7:53.

31. Chen SL, Guo LJ, Wang X. All-optical photoacoustic microscopy. Photoacoustics 2015;3:143-50.

32. Ai M, Shu W, Salcudean T, Rohling R, Abolmaesumi $\mathrm{P}$, Tang S. Design of high energy laser pulse delivery in a multimode fiber for photoacoustic tomography. Opt Express 2017;25:17713-26.

33. Beard PC, Pérennès F, Draguioti E, Mills TN. Optical fiber photoacoustic-photothermal probe. Opt Lett 1998;23:1235-7.

34. Eom J, Park SJ, Lee BH. Noncontact photoacoustic tomography of in vivo chicken chorioallantoic membrane based on all-fiber heterodyne interferometry. J Biomed Opt 2015;20:106007. 
35. Zhang C, Chen SL, Ling T, Guo L. Review of imprinted polymer microrings as ultrasound detectors: Design, fabrication, and characterization. IEEE Sens J 2015;15:3241-8.

36. Zhang C, Chen SL, Ling T, Guo LJ. Imprinted polymer microrings as high-performance ultrasound detectors in photoacoustic imaging. J Lightwave Technol 2015;33:4318-28.

37. Broadway C, Min R, Leal AG Junior, Marques C, Caucheteur C. Toward commercial polymer fiber bragg grating sensors: Review and applications. J Lightwave Technol 2019;37:2605-15.

38. Dong B, Sun C, Zhang HF. Optical Detection of Ultrasound in Photoacoustic Imaging. IEEE Trans Biomed Eng 2017;64:4-15.

39. Ho-Chiang C, Huang H, Huang CC. High-frequency ultrasound deformation imaging for adult zebrafish during heart regeneration. Quant Imaging Med Surg 2020;10:66-75.

40. Wagner JW. Optical detection of ultrasound. In: Thurston RN, Pierce AD, editors. Physical acoustics. Cambridge, MA, USA: Academic Press, 1990:201-66.

41. Winkler AM, Maslov K, Wang LV. Noise-equivalent sensitivity of photoacoustics. J Biomed Opt 2013;18:097003.

42. Ponti F, De Cinque A, Fazio N, Napoli A, Guglielmi G, Bazzocchi A. Ultrasound imaging, a stethoscope for body composition assessment. Quant Imaging Med Surg 2020;10:1699-722.

43. Liu B, Xu Z, Wang Q, Niu X, Chan WX, Hadi W, Yap $\mathrm{CH}$. A denoising and enhancing method framework for 4D ultrasound images of human fetal heart. Quant Imaging Med Surg 2021;11:1567-85.

44. Alcoz JJ, Lee CE, Taylor HF. Embedded fiber-optic FabryPerot ultrasound sensor. IEEE Trans Ultrason Ferroelectr Freq Control 1990;37:302-6.

45. Ashkenazi S, Hou Y, Buma T, O'Donnell M. Optoacoustic imaging using thin polymer etalon. Appl Phys Lett 2005;86:134102.

46. Guggenheim JA, Li J, Allen TJ, Colchester RJ, Noimark S, Ogunlade O, Parkin IP, Papakonstantinou I, Desjardins A, Zhang EZ, Beard PC. Ultrasensitive plano-concave optical microresonators for ultrasound sensing. Nat Photonics 2017;11:714-9.

47. Ansari R, Zhang EZ, Desjardins AE, Beard PC. All-optical forward-viewing photoacoustic probe for high-resolution 3D endoscopy. Light Sci Appl 2018;7:75.

48. Govindan V, Ashkenazi S. Bragg waveguide ultrasound detectors. IEEE Trans Ultrason Ferroelectr Freq Control 2012;59:2304-11.

49. Rosenthal A, Omar M, Estrada H, Kellnberger S, Razansky D, Ntziachristos V. Embedded ultrasound sensor in a silicon-on-insulator photonic platform. Appl Phys Lett 2014;104:021116.

50. Li H, Ma X, Cui B, Wang Y, Zhang C, Zhao J, Zhang Z, Tang C, Li E. Chip-scale demonstration of hybrid iii-v/ silicon photonic integration for an fbg interrogator. Optica 2017;4:692-700.

51. Yang W, Song S, Powell K, Tian X, Li L, Nguyen L, Yi $\mathrm{X}$. Etched silicon-on-insulator microring resonator for ultrasound measurement. IEEE Photonics J 2020;12:1-9.

52. Song $W$, Peng L, Guo G, Yang F, Zhu Y, Zhang C, Min C, Fang H, Zhu S, Yuan X. Isometrically Resolved Photoacoustic Microscopy Based on Broadband Surface Plasmon Resonance Ultrasound Sensing. ACS Appl Mater Interfaces 2019;11:27378-85.

53. Thomson JK, Wickramasinghe HK, Ash EA. A fabryperot acoustic surface vibration detector - application to acoustic holography. J Phys D-Appl Phys 1973;6:677-87.

54. Zhang E, Laufer J, Beard P. Backward-mode multiwavelength photoacoustic scanner using a planar Fabry-Perot polymer film ultrasound sensor for highresolution three-dimensional imaging of biological tissues. Appl Opt 2008;47:561-77.

55. Tian J, Zhang Q, Fink T, Li H, Peng W, Han M. Tuning operating point of extrinsic Fabry-Perot interferometric fiber-optic sensors using microstructured fiber and gas pressure. Opt Lett 2012;37:4672-4.

56. Cheng L, Wang C, Huang Y, Liang H, Guan BO. Silk fibroin diaphragm-based fiber-tip Fabry-Perot pressure sensor. Opt Express 2016;24:19600-6.

57. Tadayon MA, Baylor ME, Ashkenazi S. Polymeric waveguide fabry perot resonators. In: Tabor CE, Kajzar F, Kaino T, Koike Y. editors. Organic photonic materials and devices XVI. Bellingham, DC, USA: SPIE, 2014:89830W.

58. Ma J, Jin W, Ho HL, Dai JY. High-sensitivity fibertip pressure sensor with graphene diaphragm. Opt Lett 2012;37:2493-5.

59. Liao C, Liu S, Xu L, Wang C, Wang Y, Li Z, Wang Q, Wang DN. Sub-micron silica diaphragm-based fiber-tip Fabry-Perot interferometer for pressure measurement. Opt Lett 2014;39:2827-30.

60. Ma X, Cai Y, Fu B, Xu L, Ma J. Fiber optic-based laser interferometry array for three-dimensional ultrasound sensing. Opt Lett 2019;44:5852-5.

61. Chen B, Chen Y, Ma C. Photothermally tunable Fabry- 
Pérot fiber interferometer for photoacoustic mesoscopy.

Biomed Opt Express 2020;11:2607-18.

62. Liu Y, Jing Z, Li R, Zhang Y, Liu Q, Li A, Zhang C, Peng W. Miniature fiber-optic tip pressure sensor assembled by hydroxide catalysis bonding technology. Opt Express 2020;28:948-58.

63. Thathachary SV, Ashkenazi S. Toward a highly sensitive polymer waveguide fiber Fabry-Pérot ultrasound detector. J Biomed Opt 2018;23:1-12.

64. Colchester RJ, Little C, Dwyer G, Noimark S, Alles EJ, Zhang EZ, Loder CD, Parkin IP, Papakonstantinou I, Beard PC, Finlay MC, Rakhit RD, Desjardins AE. All-Optical Rotational Ultrasound Imaging. Sci Rep 2019;9:5576.

65. Köstli KP, Beard PC. Two-dimensional photoacoustic imaging by use of Fourier-transform image reconstruction and a detector with an anisotropic response. Appl Opt 2003;42:1899-908.

66. Morris P, Hurrell A, Shaw A, Zhang E, Beard P. A Fabry-Perot fiber-optic ultrasonic hydrophone for the simultaneous measurement of temperature and acoustic pressure. J Acoust Soc Am 2009;125:3611-22.

67. Zhang EZ, Beard PC. A miniature all-optical photoacoustic imaging probe. In: Oraevsky AA, Wang LV. editors. Photons plus ultrasound: Imaging and sensing. Bellingham, DC, USA: SPIE, 2011:78991F.

68. Ma X, Liu B, Cai Y, Jia D, Fu B, Xu L, Ma J. Suppression of reverberations at fiber tips for optical ultrasound sensing. Opt Lett 2020;45:2526-9.

69. Huynh N, Ogunlade O, Zhang E, Cox B, Beard P. Photoacoustic imaging using an 8-beam fabry-perot scanner. In: Oraevsky AA, Wang LV. editors. Photons Plus Ultrasound: Imaging and Sensing. Bellingham, DC, USA: SPIE, 2016:97082L.

70. Li X, Xiong K, Yang S. Large-depth-of-field opticalresolution colorectal photoacoustic endoscope. Appl Phys Lett 2019;114:163703.

71. Xiong K, Yang S, Li X, Xing D. Autofocusing opticalresolution photoacoustic endoscopy. Opt Lett 2018;43:1846-9.

72. Li C, Yang JM, Chen R, Yeh CH, Zhu L, Maslov K, Zhou Q, Shung KK, Wang LV. Urogenital photoacoustic endoscope. Opt Lett 2014;39:1473-6.

73. Dong B, Chen S, Zhang Z, Sun C, Zhang HF. Photoacoustic probe using a microring resonator ultrasonic sensor for endoscopic applications. Opt Lett 2014;39:4372-5.

74. Wang L, Lei P, Wen X, Zhang P, Yang S. Tapered fiber- based intravascular photoacoustic endoscopy for highresolution and deep-penetration imaging of lipid-rich plaque. Opt Express 2019;27:12832-40.

75. Yang Q, Duan J, Fan Z, Qu X, Xie Y, Nguyen C, Du X, Bi X, Li K, Ji X, Li D. Early Detection and Quantification of Cerebral Venous Thrombosis by Magnetic Resonance Black-Blood Thrombus Imaging. Stroke 2016;47:404-9.

76. Wu C, Zhao W, An H, Wu L, Chen J, Hussain M, Ding Y, Li C, Wei W, Duan J, Wang C, Yang Q, Wu D, Liu L, Ji $\mathrm{X}$. Safety, feasibility, and potential efficacy of intraarterial selective cooling infusion for stroke patients treated with mechanical thrombectomy. J Cereb Blood Flow Metab 2018;38:2251-60.

77. Ansari R, Zhang E, Mathews S, Desjardins AE, Beard PC. Photoacoustic endoscopy probe using a coherent fibre-optic bundle. In: Ntziachristos V, Zemp R. editors. Conference on Opto-Acoustic Methods and Applications in Biophotonics II. Bellingham, DC, USA: SPIE, 2015:953905.

78. America LIo. American national standard for safe use of lasers. Orlando, FL, USA: Laser Institute of America, 2007.

79. Huang SW, Chen SL, Ling T, Maxwell A, O'Donnell M, Guo LJ, Ashkenazi S. Low-noise wideband ultrasound detection using polymer microring resonators. Appl Phys Lett 2008;92:193509-1935093.

80. Ling T, Chen SL, Guo LJ. High-sensitivity and widedirectivity ultrasound detection using high $\mathrm{Q}$ polymer microring resonators. Appl Phys Lett 2011;98:204103.

81. Guha B, Cardenas J, Lipson M. Athermal silicon microring resonators with titanium oxide cladding. Opt Express 2013;21:26557-63.

82. Luke K, Dutt A, Poitras CB, Lipson M. Overcoming Si3N4 film stress limitations for high quality factor ring resonators. Opt Express 2013;21:22829-33.

83. Li H, Dong B, Zhang Z, Zhang HF, Sun C. A transparent broadband ultrasonic detector based on an optical microring resonator for photoacoustic microscopy. Sci Rep 2014;4:4496.

84. Rabiei P, Rao A, Chiles J, Ma J, Fathpour S. Low-loss and high index-contrast tantalum pentoxide microring resonators and grating couplers on silicon substrates. Opt Lett 2014;39:5379-82.

85. Wei H, Krishnaswamy S. Polymer micro-ring resonator integrated with a fiber ring laser for ultrasound detection. Opt Lett 2017;42:2655-8.

86. Yao B, Huang SW, Liu Y, Vinod AK, Choi C, Hoff M, Li Y, Yu M, Feng Z, Kwong DL, Huang Y, Rao Y, Duan X, Wong CW. Gate-tunable frequency combs in graphene- 
nitride microresonators. Nature 2018;558:410-4.

87. Sacher WD, Poon JK. Dynamics of microring resonator modulators. Opt Express 2008;16:15741-53.

88. Chao CY, Ashkenazi S, Huang SW, O'Donnell M, Guo LJ. High-frequency ultrasound sensors using polymer microring resonators. IEEE Trans Ultrason Ferroelectr Freq Control 2007;54:957-65.

89. Guo LJ. Nanoimprint lithography: Methods and material requirements. Adv Mater 2007;19:495-513.

90. Cheng X, Guo LJ, Fu PF. Room-Temperature, Low-Pressure Nanoimprinting Based on Cationic Photopolymerization of Novel Epoxysilicone Monomers. Adv Mater 2005;17:1419-24.

91. Martin C, Rius G, Llobera A, Voigt A, Gruetzner G, Perez-Murano F. Electron beam lithography at $10 \mathrm{kev}$ using an epoxy based high resolution negative resist. Microelectronic Engineering 2007;84:1096-9.

92. Nishikubo T, Kudo H. Recent development in molecular resists for extreme ultraviolet lithography. Journal of Photopolymer Science and Technology 2011;24:9-18.

93. Webb DJ, Surowiec J, Sweeney M, Jackson DA, Gavrilov LR, Hand JW, Zhang L, Bennion I. Miniature fibre optic ultrasonic probe. In: DePaula RP, Berthold JW III. editors. Fiber optic and laser sensors XIV. Bellingham, DC, USA: SPIE, 1996:76-80.

94. Fujisue T, Nakamura K, Ueha S. Demodulation of acoustic signals in fiber bragg grating ultrasonic sensors using arrayed waveguide gratings. Jpn J Appl Phys 2006;45:4577-9.

95. Rosenthal A, Razansky D, Ntziachristos V. High-sensitivity compact ultrasonic detector based on a pi-phase-shifted fiber Bragg grating. Opt Lett 2011;36:1833-5.

96. Liu T, Han M. Analysis of pi-phase-shifted fiber bragg gratings for ultrasonic detection. IEEE Sens J 2012;12:2368-73.

97. Wu Q, Okabe Y. Ultrasonic sensor employing two cascaded phase-shifted fiber Bragg gratings suitable for multiplexing. Opt Lett 2012;37:3336-8.

98. Shnaiderman R, Wissmeyer G, Ülgen O, Mustafa Q, Chmyrov A, Ntziachristos V. A submicrometre siliconon-insulator resonator for ultrasound detection. Nature 2020;585:372-8.

99. Zhu Y, Hu L, Liu Z, Han M. Ultrasensitive ultrasound detection using an intracavity phase-shifted fiber Bragg grating in a self-injection-locked diode laser. Opt Lett 2019;44:5525-8.

100.Homola J. Surface plasmon resonance sensors for detection of chemical and biological species. Chem Rev
2008;108:462-93.

101.Nuster R, Paltauf G, Burgholzer P. Comparison of surface plasmon resonance devices for acoustic wave detection in liquid. Opt Express 2007;15:6087-95.

102. Wang T, Cao R, Ning B, Dixon AJ, Hossack JA, Klibanov AL, Zhou Q, Wang A, Hu S. All-optical photoacoustic microscopy based on plasmonic detection of broadband ultrasound. Appl Phys Lett 2015;107:153702.

103. Yang F, Song W, Zhang C, Min C, Fang H, Du L, Wu P, Zheng W, Li C, Zhu S, Yuan X. Broadband graphenebased photoacoustic microscopy with high sensitivity. Nanoscale 2018;10:8606-14.

104. Guo G, Song W, Min C, Zhang C, Yuan X. Highsensitivity reflection-mode in vivo photoacoustic microscopy based on surface plasmon resonance sensing. In: Zhu J, Chen W, Zhang Z, Zhong M, Wang P, Qiu J. editors. 14th national conference on laser technology and optoelectronics. Bellingham, DC, USA: SPIE, 2019:1117046.

105.Song W, Guo G, Wang J, Zhu Y, Zhang C, Fang H, Min C, Zhu S, Yuan X. In Vivo Reflection-Mode Photoacoustic Microscopy Enhanced by Plasmonic Sensing with an Acoustic Cavity. ACS Sens 2019;4:2697-705.

106. Bucaro JA, Dardy HD, Carome EF. Optical fiber acoustic sensor. Appl Opt 1977;16:1761-2.

107.Park SJ, Eom J, Kim YH, Lee CS, Lee BH. Noncontact photoacoustic imaging based on all-fiber heterodyne interferometer. Opt Lett 2014;39:4903-6.

108. Ma J, Martin K, Dayton PA, Jiang X. A preliminary engineering design of intravascular dual-frequency transducers for contrast-enhanced acoustic angiography and molecular imaging. IEEE Trans Ultrason Ferroelectr Freq Control 2014;61:870-80.

109. Fei C, Ma J, Chiu CT, Williams JA, Fong W, Chen Z, Zhu B, Xiong R, Shi J, Hsiai TK, Shung KK, Zhou Q. Design of matching layers for high-frequency ultrasonic transducers. Appl Phys Lett 2015;107:123505.

110. Yin W, Xie Y, Qu Z, Liu Z. A Pseudo-3D Model for Electromagnetic Acoustic Transducers (EMATs). Appl Sci 2018;8:450.

111. Wang Y, Hu Y, Peng B, Zhou H, Zhao Y, Ma Z. Complete-noncontact photoacoustic microscopy by detection of initial pressures using a $3 \times 3$ couplerbased fiber-optic interferometer. Biomed Opt Express 2019;11:505-16.

112.Imai M, Ohashi T, Ohtsuka Y. Fiber-optic Michelson interferometer using an optical power divider. Opt Lett 1980;5:418-20. 
113.Wang Y, Li C, Wang RK. Noncontact photoacoustic imaging achieved by using a low-coherence interferometer as the acoustic detector. Opt Lett 2011;36:3975-7.

114. Park S, Rim S, Kim Y, Lee BH. Noncontact photoacoustic imaging based on optical quadrature detection with a multiport interferometer. Opt Lett 2019;44:2590-3.

115. Wang J, Peng H, Zhou P, Guo J, Jia B, Wu H. Sound source localization based on michelson fiber optic interferometer array. Opt Fiber Technol 2019;51:112-7.

116. Qiao W, Chen Z, Zhou W, Xing D. All-optical photoacoustic Doppler transverse blood flow imaging. Opt Lett 2018;43:2442-5.

117.Azuma T, Tomozawa A, Umemura SI. Observation of ultrasonic wavefronts by synchronous schlieren imaging. Japan J Appl Phys 2002;41:3308-12.

118. Nuster R, Slezak P, Paltauf G. High resolution threedimensional photoacoutic tomography with CCDcamera based ultrasound detection. Biomed Opt Express 2014;5:2635-47.

119.Pitts TA, Sagers A, Greenleaf JF. Optical phase contrast measurement of ultrasonic fields. IEEE Trans Ultrason Ferroelectr Freq Control 2001;48:1686-94.

120.Kudo N. A Simple Technique for Visualizing Ultrasound Fields Without Schlieren Optics. Ultrasound Med Biol 2015;41:2071-81.

121. Harigane S, Miyasaka R, Yoshizawa S, Umemura SI. Optical phase contrast mapping of highly focused ultrasonic fields. Japan J Appl Phys 2013;52:07HF07.

122. Barnes RA Jr, Maswadi S, Glickman R, Shadaram M. Probe beam deflection technique as acoustic emission directionality sensor with photoacoustic emission source. Appl Opt 2014;53:511-9.

123. Khachatryan E, Maswadi S, Tsyboulski DA, Barnes E, Sardar D, Oraevsky AA, Nash K, Glickman R. Optoacoustic microscopy using laser beam deflection technique. In: Oraevsky AA, Wang LV. editors. Photons plus ultrasound: Imaging and sensing. Bellingham, DC, USA: SPIE, 2014:89432T.

124. Maswadi SM, Ibey BL, Roth CC, Tsyboulski DA, Beier HT, Glickman RD, Oraevsky AA. All-optical optoacoustic microscopy based on probe beam deflection technique. Photoacoustics 2016;4:91-101.

125.Li H, Dong B, Zhang X, Shu X, Chen X, Hai R, Czaplewski DA, Zhang HF, Sun C. Disposable ultrasoundsensing chronic cranial window by soft nanoimprinting lithography. Nat Commun 2019;10:4277.

126.Xu M, Wang LV. Photoacoustic imaging in biomedicine. Rev Sci Instrum 2006;77:041101.
127. Yang JM, Maslov K, Yang HC, Zhou Q, Shung KK, Wang LV. Photoacoustic endoscopy. Opt Lett 2009;34:1591-3.

128.Huang D, Swanson EA, Lin CP, Schuman JS, Stinson WG, Chang W, Hee MR, Flotte T, Gregory K, Puliafito CA. Optical coherence tomography. Science 1991;254:1178-81.

129. Yang Y, Li X, Wang T, Kumavor PD, Aguirre A, Shung KK, Zhou Q, Sanders M, Brewer M, Zhu Q. Integrated optical coherence tomography, ultrasound and photoacoustic imaging for ovarian tissue characterization. Biomed Opt Express 2011;2:2551-61.

130. Weissleder R, Ntziachristos V. Shedding light onto live molecular targets. Nat Med 2003;9:123-8.

131. Chang Y, Hu Y, Chen Z, Xing D. Co-impulse multispectral photoacoustic microscopy and optical coherence tomography system using a single supercontinuum laser. Opt Lett 2019;44:4459-62.

132.Liu M, Drexler W. Optical coherence tomography angiography and photoacoustic imaging in dermatology. Photochem Photobiol Sci 2019;18:945-62.

133.Li L, Maslov K, Ku G, Wang LV. Three-dimensional combined photoacoustic and optical coherence microscopy for in vivo microcirculation studies. Opt Express 2009; 17:16450-5.

134.Jiao S, Jiang M, Hu J, Fawzi A, Zhou Q, Shung KK, Puliafito CA, Zhang HF. Photoacoustic ophthalmoscopy for in vivo retinal imaging. Opt Express 2010;18:3967-72.

135.Fu B, Hua Y, Xiao X, Zhu H, Sun Z, Yang C. Broadband Graphene Saturable Absorber for Pulsed Fiber Lasers at $1,1.5$, and $2 \mu \mathrm{m}$. IEEE J Sel Top Quantum Electron 2014;20:411-5.

136.Fu B, Sun J, Wang G, Shang C, Ma Y, Ma J, Xu L, Scardaci V. Solution-processed two-dimensional materials for ultrafast fiber lasers (invited). Nanophotonics 2020;9:2169-89.

137.Shang C, Zhang Y, Qin H, He B, Zhang C, Sun J, Li J, Ma J, Ji X, Xu L, Fu B. Review on wavelength-tunable pulsed fiber lasers based on d materials. Optics \& Laser Technology 2020;131:106375.

138. Erfanzadeh M, Zhu Q. Photoacoustic imaging with lowcost sources; A review. Photoacoustics 2019;14:1-11.

139. Erfanzadeh M, Kumavor PD, Zhu Q. Laser scanning laser diode photoacoustic microscopy system. Photoacoustics 2017;9:1-9.

140. Pan J, Zhang B, Liu Z, Zhao J, Feng Y, Wan L, Li Z. Microbubble resonators combined with a digital optical frequency comb for high-precision air-coupled ultrasound detectors. Photon Res 2020;8:303-10. 
141. Wildi T, Voumard T, Brasch V, Yilmaz G, Herr T. Photoacoustic dual-frequency comb spectroscopy. Nat Commun 2020;11:4164.

142. Yan M, Zhang L, Hao Q, Shen X, Qian X, Chen H, Ren $\mathrm{X}$, Zeng H. Surface-enhanced dual-comb coherent raman spectroscopy with nanoporous gold films. Laser Photonics Rev 2018;12:1800096.

143.Fu B, Zhang C, Lyu W, Sun J, Shang C, Cheng Y, Xu L. Recent progress on laser absorption spectroscopy for determination of gaseous chemical species. Appl Spectrosc Rev 2020. doi: 1080/05704928.2020.1857258.

Cite this article as: Fu B, Cheng Y, Shang C, Li J, Wang G, Zhang C, Sun J, Ma J, Ji X, He B. Optical ultrasound sensors for photoacoustic imaging: a narrative review. Quant Imaging Med Surg 2022;12(2):1608-1631. doi: 10.21037/qims-21-605
144. Friedlein JT, Baumann E, Briggman KA, Colacion GM, Giorgetta FR, Goldfain AM, Herman DI, Hoenig EV, Hwang J, Newbury NR, Perez EF, Yung CS, Coddington I, Cossel KC. Dual-comb photoacoustic spectroscopy. Nat Commun 2020;11:3152.

145.Diddams SA, Vahala K, Udem T. Optical frequency combs: Coherently uniting the electromagnetic spectrum. Science 2020;369: eaay3676.

146. Wu Q, Okabe Y, Yu F. Ultrasonic Structural Health Monitoring Using Fiber Bragg Grating. Sensors (Basel) 2018;18:3395. 